Pacific Journal of Mathematics

REPRODUCING KERNELS AND ORTHOGONAL KERNELS
TOR ANALYTIC DIFFERENTIALS ON RIEMANN SURFACES 


\title{
REPRODUCING KERNELS AND ORTHOGONAL KERNELS FOR ANALYTIC DIFFERENTIALS ON RIEMANN SURFACES
}

\author{
Georges G. WeILL
}

Introduction. In the case of plane regions of finite connectivity, there exist (Bergman [2]) some kernel functions which possess reproducing or orthogonal properties with respect to the space of analytic exact differentials. Such Bergman kernels can be defined in a constructive way, and are related to some derivatives of the Neumann function for the region. Starting with the Green's function, one may construct the corresponding Bergman kernels for the space of analytic differentials in the region.

On the other hand, by Hilbert space methods one may prove the existence of corresponding kernels on arbitrary Riemann surfaces (Ahlfors and Sario [1]) = these methods are of the nonconstructive type.

In this paper we shall construct actually such kernels for the space of analytic differentials on an arbitrary Riemann surface $W$. We shall first establish the relationship between the kernels and some principal functions in the case of a compact bordered $\bar{W}$. The principal functions solve some well defined boundary value problems on $\bar{W}$. By a canonical exhaustion by regular regions (with compact bordered closures) the results are extended to an open Riemann surface $W$.

Chapter I is preliminary in nature and contains important theorems as well as definitions used in the sequel. It is a brief survey of the theory of principal functions and of the theory of differentials on Riemann surfaces (Ahlfors and Sario [1]).

In Chapter II, we introduce the concept of abstract reproducing and orthogonal kernels (Bergman [2], Schiffer [5]). We prove a uniqueness theorem for the reproducing kernel corresponding to a closed subspace $\Gamma_{a}$ of $\Gamma_{a}$, the space of analytic differentials on a Riemann surface $W$, and for the related orthogonal kernels with a given analytic singularity. We then determine some functionals which are extremalized by reproducing and orthogonal kernels. In particular, the reproducing kernel $k_{0}(z, \zeta) d z$ for $\Gamma_{\alpha}$ minimizes the expression

$$
\|a d z\|^{2}-2 \operatorname{Rea}(\zeta)
$$

among all differentials $a d z \in \Gamma_{\alpha}$, and the kernel $h_{0}(z, \zeta) d z$ with singularity

Received August 21, 1961. The author wishes to express his most sincere gratitude to Professor Leo Sario for his suggesting this investigation and for his guidance during its progress. 
$d z /(z-\zeta)^{2}$ orthogonal to the class $\Gamma_{a e}$ of analytic exact differentials minimizes the functional

$$
\frac{i}{2} \int_{\beta} f \overline{d f}
$$

among all differentials of functions analytic on $W$ except for the singularity $-(1 /(z-\zeta))$.

Such extremal properties suggest some close relationship between the kernels and the principal functions on $W$.

In Chapter III, the case of planar surfaces is investigated in details. First we study $\Gamma_{a e}$. It is shown that for a compact bordered surface $\bar{W}$, with principal analytic functions $P_{0}$ and $P_{1}$ corresponding to the singularity $1 /(z-\zeta)$ the following relations hold:

$$
\begin{aligned}
& \frac{d}{d z}\left(\frac{P_{1}-P_{0}}{2}\right) d z=-\widetilde{k}_{0} d z \\
& \frac{d}{d z}\left(\frac{P_{1}+P_{0}}{2}\right) d z=-\widetilde{h}_{0} d z
\end{aligned}
$$

where $\tilde{k}_{0} d z$ is the reproducing kernel for $\Gamma_{a e}$, and $\tilde{h}_{0} d z$ is the orthogonal kernel corresponding to the singularity $d z /(z-\zeta)^{2}$.

The preceding properties are shown there to hold for arbitrary planar Riemann surfaces.

Moreover, analogous properties for other kind of kernels enable us to prove relations between the principal functions $P_{i m}$ corresponding to the singularity $1 /(z-\zeta)^{m}, m>1$ and the principal functions $P_{i}$ corresponding to the singularity $1 /(z-\zeta)$.

We then complete the study of the kernels for $\Gamma_{a}$. We show the relationship between Bergman kernels and principal functions in the compact bordered case, and then construct directly reproducing and orthogonal kernels for the orthogonal complement $\Gamma_{a m}$ of $\Gamma_{a e}$.

In Chapter IV we extend the result of Chapter III to Riemann surfaces of nonzero genus. In particular, starting from the harmonic principal functions $p_{0}$ and $p_{1}$ for a nonplanar compact bordered $\bar{W}$, we construct reproducing and orthogonal kernels for $\Gamma_{a e}$. For an open Riemann surface $W$, the corresponding kernels are constructed, using a canonical exhaustion of $W$ by regular regions, with compact bordered closures. To obtain the kernel for $\Gamma_{a}$, it is then only necessary to get kernels for $\Gamma_{a s}$, the space of analytic Schottky differentials, which is the orthogonal complement of $\Gamma_{a e}$. We construct the kernels in the compact bordered case by considering the double $\hat{W}$ of $\bar{W}$ and use the theory of differentials on closed surfaces. By a limiting process (canonical exhaustion) we extend our results to an open Riemann surface $W$. 


\section{Chapter I. PRELIMINARIES}

\section{Principal Functions.}

1A. We summarize here the theory of principal functions on open Riemann surfaces. For a more complete discussion we refer to [1]. Let $W$ be an open Riemann surface. At a finite number of points $\zeta_{j} \in W$ there are given singularities of the form:

$$
s^{(j)}=\operatorname{Re} \sum_{n=1}^{\infty} b_{n}^{(j)}\left(z-\zeta_{j}\right)^{-n}+c^{(j)} \log \left|z-\zeta_{j}\right|
$$

where the $c^{(j)}$ are real and subject to the condition $\sum_{j} c^{(j)}=0$. Then there exist functions harmonic on $W$ except for the singularity $s^{(j)}$ at $\zeta_{j}$. The "principal" functions are defined in the following way: if $\left\{\Omega_{n}\right\}$ is a canonical exhaustion of $W$, then $p_{0}$ is the limit of such functions on $\Omega_{n}$ which have vanishing normal derivatives on the boundary $\beta\left(\Omega_{n}\right)$ of $\Omega_{n}$ and if $\mathscr{P}$ is a partition of the ideal boundary $\beta$ of $W$, then $(\mathscr{P})_{p_{1}}$ is the limit of such functions on $\Omega_{n}$, which are constant on each part of $\beta\left(\Omega_{n}\right)$ associated with $\mathscr{P}$ and whose conjugate periods along each part is zero. The functions $p_{0}$ and $(\mathscr{P})_{p_{1}}$ are unique save for an additive constant.

1B. Let $p$ be a function harmonic on $W$ except for a finite number of singularities $s^{(j)}$. Near $\zeta_{j}, p-s^{(j)}$ has the following expansion:

$$
p-s^{(j)}=a_{0}^{(j)}+a_{1}^{(j)}\left(z-\zeta_{j}\right)+a_{2}^{(j)}\left(z-\zeta_{j}\right)^{2}+\cdots
$$

We introduce the notation

$$
C(p)=2 \pi R e\left\{\sum_{j}\left[c^{(i)} a_{0}^{(j)}-\sum_{n=1}^{\infty} n b_{n}^{(j)} a_{n}^{(j)}\right]\right\}
$$

and set $C_{0}, C_{1}$ for $C\left(p_{0}\right), C\left(p_{1}\right)$ respectively

Let $\Omega$ be a regular subregion of $W$ with boundary $\beta(\Omega)$, containing all $\zeta_{j}$ in its inside. We set

$$
B_{\Omega}(p)=\int_{\beta(\Omega)} p d p^{*}
$$

and define $D_{\Omega}\left(p-\sum_{j} s^{(i)}\right)$ as the Dirichlet integral of $p-\sum_{j} s^{i}$ over $\Omega$.

Now let

$$
B(p)=\lim _{\Omega \rightarrow W} \int_{\beta(\Omega)} p d p^{*}
$$


and

$$
D\left(p-\sum s^{(j)}\right)=\lim _{\Omega \rightarrow W} D_{\Omega}\left(p-\sum_{j} s^{(j)}\right)
$$

We have the following

THEOREM. The function $\left(p_{0}+p_{1}\right) / 2$ minimizes the functional $B(p)$ in the class of all functions $p$ with singularities $\sum_{j} s^{(j)}$ whose conjugate periods vanish over all dividing cycles associated with the partition $\mathscr{P}$. (Here $p_{1}$ stands for $\left.\mathscr{P}\left(p_{1}\right)\right)$. Explicitly the formula:

$$
B(p)=\frac{1}{4}\left(C_{0}-C_{1}\right)+D\left(p-\frac{p_{0}+p_{1}}{2}\right)
$$

which is valid for such functions shows that the minimum is $\left(C_{0}-C_{1}\right) / 4$ and that the deviation from the minimum is measured by $D\left(p-\left(\left(p_{0}+p_{1}\right) / 2\right)\right)$.

Let now $q=p_{0}-p_{1}$. The harmonic function $q$ satisfies the following theorem:

THEOREM. For regular admissible functions the expression

$$
D(u)+4 \pi\left(u\left(\zeta_{1}\right)-u\left(\zeta_{2}\right)\right)
$$

attains its minimum when $u=q=p_{0}-p_{1}$. Here $p_{0}$ and $p_{1}$ are the principal functions corresponding to the singularity $\log \left|z-\zeta_{1}\right|-$ $\log \left|z-\zeta_{2}\right|$. Similarly,

$$
D(u)-\left.\frac{4 \pi}{(n-1) !} R e \frac{\partial^{n} u}{\partial z^{n}}\right|_{z=\zeta}
$$

is minimized by the function $q=p_{0}-p_{1}$ which corresponds to the singularity $\operatorname{Re}\left(1 /(z-\zeta)^{n}\right)$.

In both cases the minimum is $-D(q)$ and the deviation from the minimum is $D(u-q)$.

1C. We shall make use of the following result

THEOREM. Let $\Omega$ and $\Omega^{\prime}$ be two regular subregions of a Riemann surface $W$, such that $\Omega^{\prime} \supset \Omega$; let

$$
p_{i \Omega} \quad \text { and } \quad p_{i \Omega^{\prime}} \quad i=(0,1)
$$

be the principal functions corresponding respectively to $\Omega$ and $\Omega^{\prime}$; let

$$
D_{\Omega}\left(p_{i, \Omega^{\prime}}-p_{i \Omega}\right) \quad i=(0,1)
$$

be the Dirichlet integral of $p_{i \Omega^{\prime}}-p_{i \Omega}$ extended over $\Omega$, then 


$$
\lim _{\Omega \rightarrow W} D_{\Omega}\left(p_{i \Omega^{\prime}}-p_{i \Omega}\right)=0 \quad i=(0,1)
$$

1D. Previous results may be specialized to the case of planar Riemann surfaces. By definition, every cycle on a planar surface $W$ is dividing. For this reason it is natural to consider the canonical partition $\mathscr{Q}$ for the boundary $\beta$. Accordingly, the admissible functions, in the planar case, have all conjugate periods equal to zero. This means that $P=$ $p+i p^{*}$ is an analytic (single-valued) function. The functions $p$ are supposed to have a given singularity $s$ and if $p^{*}$ is to be single valued, we require that $s$ has likewise a single-valued conjugate function $s^{*}$. We then shall not consider singularities with logarithmic terms. We denote by $P_{0}$ and $P_{1}$ the analytic functions which correspond to the principal functions $p_{0}$ and $p_{1}$. They are determined up to additive complex constants. If we denote by $E$ the area of the complement of the range of $P$ and by $a$ the coefficient in the expansion.

$$
P=\frac{1}{z-\zeta}+a(z-\zeta)+\cdots,
$$

we have the following result:

THEOREM. The function $\left(P_{0}+P_{1}\right) / 2$ maximizes $E$ in the class of all normalized univalent mappings. In this case $E=(\pi / 2)\left[a\left(P_{0}\right)-a\left(P_{1}\right)\right]$.

1E. Consider now the analytic function $Q=P_{0}-P_{1}$. The properties of $Q$ are summarized in the following theorem.

THEOREM. The function $Q$ minimizes the expression $D(U)-$ $4 \pi R e a(U)$ in the class of all analytic functions $U=u+i u^{*}$ on $W$. Moreover, $a(Q)$ is nonnegative and $2 \pi a(Q)=D(Q)=E$ where $E$ denotes the complementary area associated with the mapping $P_{0}+P_{1}$.

\section{Differentials on Riemann surfaces.}

2A. We shall suppose the reader acquainted with the definitions and orthogonal decompositions of the Hilbert space $\Gamma$ of square integrable differentials on an open Riemann surface. (See, for instance, [1] Chapter $V)$. We merely recall some results about some subspaces of $\Gamma$ and state the theorems on singular differentials which we shall use below.

2B. Schottky differentials.

Let $\bar{W}$ be a bordered Riemann surface, and $\hat{W}$ its double. The whole class of differentials on $W$ which have a harmonic extension to $\hat{W}$ can be shown to be identical with the direct $\operatorname{sum} \Gamma_{h 0}(W)+\Gamma_{h 0}^{*}(W)$. Such differentials are called Schottky differentials. 
If now one considers, on an arbitrary open surface all differentials which can be approximated in the sense of the norm by Schottky differentials, i.e. by differentials in $\Gamma_{h 0}(\Omega)+\Gamma_{h 0}^{*}(\Omega)(\Omega$ being a regular subregion of $W$ ), the corresponding subspace $\Gamma_{s}$ can be shown to be identical with the closure of $\Gamma_{h 0}+\Gamma_{h 0}^{*}$.

The consideration of Schottky differentials leads to the following decomposition:

Theorem. On any Riemann surface

$$
\Gamma_{h}=\Gamma_{s}+\Gamma_{a e}+\overline{\Gamma_{a e}}
$$

where $\Gamma_{a e}$ is the space of exact analytic differentials, $\overline{\Gamma_{a e}}$ the space of exact antianalytic differentials.

2C. Analytic differentials.

If our attention is restricted to analytic differentials, writing $\Gamma_{a s}=$ $\Gamma_{a} \cap \Gamma_{s}$ we obtain the following decomposition:

$$
\Gamma_{a}=\Gamma_{a e}+\Gamma_{a s} .
$$

In the case of a compact bordered $\bar{W}$, the following result holds: An analytic Schottky differential on $\bar{W}$ can be written as the sum of an analytic differential which is real along the boundary and one which is purely imaginary along the boundary.

On an open surface $W$, an analytic Schottky differential $\phi$ is by definition a limit of analytic Schottky differentials $\omega_{a s \Omega}$. It follows that:

$$
\phi=\lim _{\Omega \rightarrow W}\left(\phi_{1, \Omega}+i \phi_{2, \Omega}\right)
$$

where $\phi_{1 \Omega}$ and $\phi_{2 \Omega}$ are real along $\beta(\Omega)$.

The following theorem states an important decomposition property of analytic differentials:

THEOREM. Any analytic differential $\phi_{a}$ has a unique representation in the form:

$$
\phi_{a}=\phi_{a s e}+\omega_{h m}+i \omega_{h m}^{*}
$$

where $\phi_{a s e}$ is semi exact analytic and $\omega_{h m}$ is a harmonic measure.

If we call a differential of the form $\omega_{h m}+i \omega_{h m}^{*}$ an analytic measure and $\Gamma_{a m}$ the space of analytic measures we can write:

$$
\Gamma_{a}=\Gamma_{a s e}+\Gamma_{a m} .
$$


2D. Singular differentials.

Consider a point $p_{0}$ on a Riemann surface $W$. We say that a differential $\theta_{0}$ defines a singularity at $p_{0}$ if it is defined in a punctured neighborhood of $p_{0}$. We speak of an analytic singularity if $\theta_{0}$ is an analytic differential in a punctured neighborhood of $p_{0}$.

A differential of the second kind is a differential whose singularities are poles with vanishing residues. We can consider one pole at a time, hence it is sufficient to study the case of a single pole

$$
\frac{d z}{(z-\zeta)^{m+2}} \quad m \geqq 0
$$

where $z$ is a local variable with range $|z|<1$ and $\zeta$ the value of $z$ at $p_{0}$. The following theorem summarizes the study of such differentials.

THEOREM. With every singularity of the form $(z-\zeta)^{-m-2} d z, m \geqq 0$, one can associate two differentials

$$
\begin{array}{ll}
\phi_{m}=h_{m}(z, \zeta) d z & \text { analytic except for the given singularity } \\
\psi_{m}=k_{m}(z, \zeta) d z & \text { analytic. }
\end{array}
$$

The differentials $\phi_{m}$ and $\psi_{m}$ are connected by

$$
\begin{aligned}
& h_{m}(z, \zeta)=\frac{1}{(m+1) !} \frac{d^{m}}{d z^{m}} h_{0}(z, \zeta) \\
& \overline{k_{m}(z, \zeta)}=\frac{1}{(m+1) !} \frac{d^{m}}{d \zeta^{m}} \overline{k_{0}(z, \zeta)}
\end{aligned}
$$

and $h_{0}, k_{0}$ satisfy symmetry relations:

$$
h_{0}(z, \zeta)=h_{0}(\zeta, z), \quad k_{0}(z, \zeta)=\overline{k_{0}(\zeta, z)}
$$

The differentials $\psi_{m}$ have the reproducing property:

$$
\left(a, \psi_{m}\right)=\frac{2 \pi}{(m+1) !} a^{(m)}(\zeta)
$$

for all $\alpha=a d z \in \Gamma_{a}$ while the $\phi_{m}$ satisfy $\left(\alpha, \phi_{m}\right)=0$, provided that the inner product is interpreted as a Cauchy limit.

2E. Differentials and chains.

In the Laurent development

$$
\sum_{n=1}^{\infty} b_{n}(z-\zeta)^{-n} d z
$$

of a singular differential $\theta, b_{1}$ is called the residue at $\zeta$. It can be shown that in order that there exists a closed differential $\theta$ with compact support and given singularities, it is necessary that the sum of the 
residues be 0 . The simplest case is represented by a singularity $\left(1 /\left(z-\zeta_{1}\right)-\right.$ $\left.1 /\left(z-\zeta_{2}\right)\right) d z$ where $\zeta_{1}$ and $\zeta_{2}$ are points in the same parametric disc $\Delta$ mapped on $|z|<1$. Results of the study of such differentials are summarized in the following theorem

THEOREM. To each finite chain $c$ one can assign two differentials

$$
\phi(c)=h(z, c) d z
$$

analytic except for simple poles with residues equal to the coefficients in $\partial c$ and

$$
\psi(c)=k(z, c) d z
$$

analytic. One has:

$$
\begin{aligned}
& h(z, c)=\int_{c} h_{0}(z, \zeta) d \zeta \\
& k(z, c)=\int_{c} k_{0}(z, \zeta) d \bar{\zeta}
\end{aligned}
$$

and $(\alpha, \psi)=-(\alpha, \phi)=2 \pi \int_{c} \alpha$ for analytic $\alpha$.

2F. Differentials and periods.

If $c$ is a finite cycle, $\phi(c), \psi(c)$ are all regular. Results about this case are stated below:

THEOREM. If $c$ is a finite cycle $\phi(c)=-\psi(c)$ and the reproducing differential $\sigma(c)$ corresponding to the cycle $c$ is $\sigma(c)=(1 / \pi) \operatorname{Im} \phi(c)$. The periods of $\sigma(c)$ are integers.

2G. One can strengthen Theorems $2 \mathrm{D}$ and $2 \mathrm{E}$ by requiring the associated differentials to be semi-exact. In that case we denote them by $\tilde{\phi}_{m}, \widetilde{\psi}_{m}, \tilde{\phi}(c), \widetilde{\psi}(c)$.

It can be shown that $\tilde{\phi}$ and $\tilde{\psi}$ have the same reproducing properties as $\phi$ and $\psi$, but only with respect to semi-exact analytic differentials. Since $\widetilde{\phi}_{m}$ and $\widetilde{\psi}_{m}, \widetilde{\phi}(c)$ and $\widetilde{\psi}(c)$ are themselves semi exact, Theorems 2D, $2 \mathrm{E}$ remain valid with modified notations, except that the differentials $\alpha$ must be assumed semi exact.

2H. Remark on integration by parts.

Let $\omega \in \Gamma, d f \in \Gamma_{e}$. If $\partial \bar{W}$ denotes the boundary of a bordered surface a useful formula for integration by parts is:

$$
\int_{W}(d f) \omega=\int_{\partial \bar{W}} f \omega-\int_{W} f d \omega .
$$


We plan to extend such a formula to differentials with singularities. Let $\omega$ be a closed differential with an analytic singularity at $z=\zeta$. Let $h$ be an admissible local homeomorphism representing a neighborhood of $\zeta$ onto the disk $|z|<1$. Let $|z|<r<1$ be a disk whose inverse image under $h$ will be called $\Delta$. We denote by $\alpha$ the boundary of $\Delta$. The previous formula for integration by parts yields:

$$
\int_{W-4}(d f) \omega=\int_{\partial \bar{w}-\alpha} f \omega .
$$

But now in $\Delta$, if we consider $\int_{\Delta}(d f) \omega$ as a Cauchy limit, the following relation holds:

$$
\int_{\Delta}(d f) \omega=\int_{\alpha} f \omega
$$

and by addition we get the formula

$$
\int_{W}(d f) \omega=\int_{\partial \bar{W}} f \omega .
$$

Notice that on $W, \partial \bar{W}-\alpha$ bounds the 2-chain $W-\Delta$, which implies that $\partial \bar{W}$ and $\alpha$ are homologous on $W$. The last integral depends only on the homology class of $\partial \bar{W}$, hence can be transferred to $\alpha$.

Chapter II. GENERAL DEFINITIONS AND FUNDAMENTAL PROPERTIES OF REPRODUCING AND ORTHOGONAL KERNELS

We shall define here some particular kernels and derive some of their characteristic extremal properties.

\section{General Properties.}

1A. Definition. Let $\Gamma_{\alpha}$ be a subspace of $\Gamma_{a}$. A differential $k_{0}(z, \zeta) d z$ defined globally will be called a reproducing kernel for $\Gamma_{\alpha}$ if

1. $k_{0}(z, \zeta) d z \in \Gamma_{\alpha}$.

2. for $a(z) d z \in \Gamma_{\alpha}$, the inner product $\left(a(z) d z, k_{0}(z, \zeta) d z\right)=a(\zeta)$.

More generally we shall consider $m$-kernels according to the following definition:

Definition. A globally defined differential $k_{m}(z, \zeta) d z$ is an $m$-kernel for $\Gamma_{a} \subset \Gamma_{a}$ whenever:

1. $k_{m}(z, \zeta) \in \Gamma_{\alpha}$

2. for $a(z) d z \in \Gamma_{\alpha},\left(\alpha(z) d z, k_{m}(z, \zeta) d z\right)=a^{(m)}(\zeta) \quad$ where $\quad a^{(m)}(\zeta)=$ $\left.\frac{d^{m} a(z)}{d z^{m}}\right|_{z=\zeta}$. 
1B. We introduce now some kernels with singularities. Given an analytic singularity $t(z, \zeta) d z$, one may consider differentials which are elements of $\Gamma_{a}$ except for the singularity $t(z, \zeta) d z$.

Definition. A differential $h_{t}(z, \zeta) d z$ is an orthogonal kernel for $\Gamma_{a} \subset \Gamma_{a}$, corresponding to the analytic singularity $t(z, \zeta) d z$ if the following requirements are fulfilled.

1. $h_{t}(z, \zeta)$ is an element of $\Gamma_{\alpha}$ except for the singularity $t(z, \zeta) d z$

2. for $a(z) d z \in \Gamma_{\alpha},\left(a(z) d z, h_{t}(z, \zeta) d z\right)=0$.

In particular we shall call $h_{m}(z, \zeta) d z$, the orthogonal kernel corresponding to the singularity $d z /(z-\zeta)^{m+2}$ for $m \geqq 0$.

1C. Now we can easily prove uniqueness theorems.

THEOREM. When there exists a reproducing kernel for $\Gamma_{\alpha}$, it is unique.

Proof. Let $k_{0}(z, \zeta) d z$ and $k_{0}^{\prime}(z, \zeta) d z$ be two different reproducing kernels for $\Gamma_{\alpha}$. Then

$$
\begin{aligned}
0 \leqq & \left\|k_{0}(z, \zeta) d z-k_{0}^{\prime}(z, \zeta) d z\right\|^{2} \\
= & \left(k_{0}(z, \zeta) d z-k_{0}^{\prime}(z, \zeta) d z, k_{0}(z, \zeta) d z\right) \\
& \quad-\left(k_{0}(z, \zeta) d z-k_{0}^{\prime}(z, \zeta) d z, k_{0}^{\prime}(z, \zeta) d z\right) \\
= & k_{0}(\zeta, \zeta) d z-k_{0}^{\prime}(\zeta, \zeta) d z-k_{0}(\zeta, \zeta) d z+k_{0}^{\prime}(\zeta, \zeta) d z=0 .
\end{aligned}
$$

Therefore $k_{0}(z, \zeta) d z=k_{0}^{\prime}(z, \zeta) d z$.

1D. An analogous argument proves the next statement.

THEOREM. When there exists an m-kernel for $\Gamma_{\alpha}$ it is unique.

1E. Correspondingly the following uniqueness theorem holds for $h_{t}(z, \zeta) d z$.

THEOREM. When there exists for $\Gamma_{a}$ an orthogonal kernel $h_{t}(z, \zeta) d z$ with singularity $t(z, \zeta) d z$, it is unique.

Proof. If $h_{t} d z$ and $h_{t}^{\prime} d z$ are two different orthogonal kernels corresponding to the same singularity, $h_{t} d z-h_{t}^{\prime} d z$ is regular and orthogonal to $\Gamma_{\alpha}$. But $h_{t} d z-h_{t}^{\prime} d z \in \Gamma_{\alpha}$. Therefore $h_{t} d z-h_{t}^{\prime} d z=0$, which proves uniqueness. 


\section{Extremal Properties.}

2A. We shall prove some extremal properties which characterize reproducing and orthogonal kernels.

THEOREM. Among all $a(z) d z \in \Gamma_{\alpha}$, such that $a^{(m)}(\zeta)=1,\left(k_{m}(z, \zeta) /\right.$ $\left.k_{m}^{(m)}(\zeta, \zeta)\right) d z$ has minimal norm.

Proof. By Schwarz's inequality:

$$
\left|\left(a(z) d z, k_{m}(z, \zeta) d z\right)\right|^{2} \leqq\|a d z\|^{2}\left\|k_{m}(z, \zeta) d z\right\|^{2} .
$$

But

$$
\begin{gathered}
\left(a(z) d z, k_{m}(z, \zeta) d z\right)=a^{(m)}(\zeta)=1 \\
\left\|k_{m} d z\right\|^{2}=\left(k_{m}(z, \zeta) d z, k_{m}(z, \zeta) d z\right)=k_{m}^{(m)}(\zeta, \zeta) .
\end{gathered}
$$

Therefore: $\|a(z) d z\|^{2} \geqq 1 / k_{m}^{(m)}(\zeta, \zeta)$ with equality only for $a(z) d z=$ $\lambda k_{m}(z, \zeta) d z$. Due to the normalization, $\lambda=k_{m}^{(m)}(\zeta, \zeta)$ and therefore $\left(k_{m}(z, \zeta) / k_{m}^{(m)}(\zeta, \zeta)\right) d z$ has minimal norm among all $a(z) d z \in \Gamma_{\alpha}$ such that $a^{(m)}(\zeta)=1$.

$2 \mathrm{~B}$. The following theorem introduces a functional which is extremalized by $k_{m}(z, \zeta) d z$.

THEOREM. Among all differentials $a(z) d z \in \Gamma_{\alpha}, k_{m}(z, \zeta)$ minimizes the expression $\|a d z\|^{2}-2 \operatorname{Rea}^{(m)}(\zeta)$. The minimum is $-k_{m}^{(m)}(\zeta, \zeta)$ and the deviation from the minimum is measured by $\left\|a d z-k_{m} d z\right\|^{2}$.

Proof. Let $a(z) d z$ be an element of $\Gamma_{\alpha}, k_{m}(z, \zeta) d z$ be the $m$ kernel for $\Gamma_{\alpha}$. Then:

$$
\begin{aligned}
\left\|a(z) d z-k_{m}(z, \zeta) d z\right\|^{2}=\|a d z\|^{2}+\left\|k_{m} d z\right\|^{2} & -\left(\alpha(z) d z, k_{m}(z, \zeta) d z\right) \\
& -\left(k_{m}(z, \zeta) d z, a(z) d z\right) .
\end{aligned}
$$

The last equation can be rewritten as:

$$
\|a(z) d z\|^{2}-2 \operatorname{Re}\left(a(z) d z, k_{m}(z, \zeta) d z\right)=\left\|a d z-k_{m} d z\right\|^{2}-\left\|k_{m} d z\right\|^{2} .
$$

But $\operatorname{Re}\left(a(z) d z, k_{m}(z, \zeta) d z\right)=\operatorname{Rea}^{(m)}(\zeta)$. Therefore:

$$
\|a(z) d z\|^{2}-2 \operatorname{Re} a^{(m)}(\zeta)=\left\|a d z-k_{m} d z\right\|^{2}-\left\|k_{m} d z\right\|^{2}
$$

which establishes the extremal property. The minimum is 


$$
-\left\|k_{m} d z\right\|^{2}=-\left(k_{m}(z, \zeta) d z, k_{m}(z, \zeta) d z\right)=-k_{m}^{(m)}(\zeta, \zeta) .
$$

2C. An important extremal property can be proved for the kernel $h_{m}(z, \zeta) d z$ orthogonal to the class $\Gamma_{a e}$, among the class of differentials of functions analytic on $W$ except for the singularity $-1 /(m+1)(z-\zeta)^{m+1}$, $m \geqq 0$. Let us call $\Gamma_{a e}^{(m)}$ such a class. Clearly $h_{m}(z, \zeta) d z$ is the differential of such a function $H_{m}(z, \zeta)$. In other words $h_{m}(z, \zeta) d z \in \Gamma_{a e}^{(m)}$.

Let $\bar{W}$ be now a compact bordered Riemann Surface. Define a surface $W^{\prime}$ obtained by deleting from $W$ the inverse image $\Delta$ of the disk $|z|<r<1$, where $|z|<1$ is the image of $\Delta$. We shall suppose that $\zeta$ lies in $\Delta$.

If $\alpha$ denotes the boundary of $\Delta$, and $\beta$ the boundary of $\bar{W}$ we shall write for $f$ analytic on $W^{\prime}$ :

$$
\begin{aligned}
& B(f)=\frac{i}{2} \int_{\beta} f \overline{d f} \\
& A(f)=\frac{i}{2} \int_{\beta} f \overline{d f}
\end{aligned}
$$

Applying Green's formula to $W^{\prime}$ we get

$$
\|d f\|^{2}=2(B(f)-A(f))
$$

We are now ready to prove the following theorem.

Theorem. The kernel $h_{m}(z, \zeta) d z$ orthogonal to the class $\Gamma_{a e}$ minimizes the functional $B(a)$ among all differentials $d a=a^{\prime}(z) d z$ in $\Gamma_{a e}^{(m)}$.

Proof. We first consider the compact bordered case. Let $W^{\prime}$ be the surface obtained by the method mentioned above from a compact bordered $\bar{W}$. Let $h_{m} d z=d H_{m}$ be the orthogonal kernel for the class $\Gamma_{a e}$, corresponding to the singularity $d z /(z-\zeta)^{m+2} m \geqq 0$. For each $d a=a^{\prime}(z) d z$ we have:

$$
\left\|a^{\prime} d z-h_{m} d z\right\|_{W^{\prime}}^{2}=\left\|a^{\prime} d z\right\|_{W^{\prime}}^{2}+\left\|h_{m} d z\right\|_{W^{\prime}}^{2}-2 \operatorname{Re}\left(a^{\prime} d z, n_{m} d z\right)_{W^{\prime}} .
$$

But

$$
\begin{aligned}
& \left\|a^{\prime} d z\right\|_{W^{\prime}}^{2}=2(B(a)-A(a)) \\
& \left\|h_{m} d z\right\|_{W^{\prime}}^{2}=2\left(B\left(H_{m}\right)-A\left(H_{m}\right)\right) .
\end{aligned}
$$

Therefore:

$$
\begin{aligned}
& \left\|a^{\prime} d z-h_{m} d z\right\|_{W^{\prime}} \\
& \quad=\left\|a^{\prime} d z\right\|_{W^{\prime}}^{2}-\left\|h_{m} d z\right\|_{W^{\prime}}^{2}-2 \operatorname{Re}\left(a^{\prime} d z-h_{m} d z, h_{m} d z\right)_{W^{\prime}} \\
& \quad=2[B(a)-A(a)]-2\left[B\left(H_{m}\right)-A\left(H_{m}\right)\right]-2 \operatorname{Re}\left(a^{\prime} d z-h_{m} d z, h_{m} d z\right)_{W^{\prime}} .
\end{aligned}
$$


But as $r \rightarrow 0, A(a) \rightarrow 0$ and $A\left(H_{m}\right) \rightarrow 0$ as Cauchy limits. Moreover, by orthogonality $\left(\left(a^{\prime}-h_{m}\right) d z, h_{m} d z\right)_{\bar{w}}=0$. Therefore:

$$
\begin{aligned}
\left\|a^{\prime} d z-h_{m} d z\right\|_{\bar{W}}^{2} & =2 B(a)-2 B\left(H_{m}\right)-2 R e\left(a^{\prime} d z-h_{m} d z, h_{m} d z\right)_{\bar{W}} \\
& =2 B(a)-2 B\left(H_{m}\right)
\end{aligned}
$$

by the orthogonal property of $h_{m} d z$ over $\Gamma_{a e}$. Hence

$$
B(\alpha)=B\left(H_{m}\right)+\frac{1}{2}\left\|a^{\prime} d z-h_{m} d z\right\|_{\bar{W}}^{2}
$$

which proves the theorem.

To extend the extremal property to open Riemann surfaces $W$, we exhaust $W$ by canonical regions $\Omega$.

For $\Omega^{\prime} \supset \Omega, H_{m \Omega^{\prime}}$ is a competing function hence:

$$
B_{\Omega}\left(H_{m \Omega^{\prime}}\right)=B_{\Omega}\left(H_{m \Omega}\right)+\frac{1}{2}\left\|d H_{m \Omega^{\prime}}-d H_{m \Omega}\right\|_{\Omega}^{2}
$$

and clearly $B_{\Omega^{\prime}}\left(H_{m \Omega^{\prime}}\right) \geqq B_{\Omega}\left(H_{m \Omega^{\prime}}\right)$. Therefore

$$
B_{\Omega^{\prime}}\left(H_{m \Omega^{\prime}}\right) \geqq B_{\Omega}\left(H_{m \Omega}\right)
$$

which shows that $B_{\Omega}\left(H_{m \Omega}\right)$ is nondecreasing. If there exists a function $P$ on $W$, analytic except for the singularity $-1 /(m+1)(z-\zeta)^{m+1}$ and such that $\lim _{\Omega \rightarrow W} B_{\Omega}(P)<\infty$, then the functional $B_{\Omega}\left(H_{m \Omega}\right)$ has a finite limit when $\Omega \rightarrow W$. Consequently $(1 / 2)\left\|d H_{m \Omega^{\prime}}-d H_{m \Omega}\right\|^{2}=D_{\Omega}\left(H_{m \Omega^{\prime}}-\right.$ $H_{m \Omega}$ ), where $D_{\Omega}$ is the Dirichlet integral extended over $\Omega$, has limit zero when $\Omega \rightarrow W$. It follows in the customary way that there exists then in $\Gamma_{a e}^{(m)}$ an orthogonal kernel $d H_{m}=\lim _{\Omega \rightarrow W} d H_{m \Omega}$.

In the case of a planar surface $P_{0}$ or $P_{1}$ can play the part of such a function $P$.

2D. We shall now consider particular types of kernels corresponding to the set of chains on a Riemann surface.

Let first $\zeta_{1}$ and $\zeta_{2}$ be two points on a Riemann surface, and $c$ a path joining $\zeta_{1}$ to $\zeta_{2}$. We say that $k(z, c) d z$ is a reproducing kernel attached to the path $c$ for the class $\Gamma_{\alpha}$ whenever:

1. $k(z, c) d z \in \Gamma_{\infty}$

2. for any $a(z) d z \in \Gamma_{\alpha},(a(z) d z, k(z, c) d z)=\int_{c} a d z$.

The uniqueness proof is analogous to the proof of Theorem $1 \mathrm{C}$. We 
shall prove an extremal property for $k(z, c) d z$.

THEOREM. The kernel $k(z, c) d z$ attached to the path $c$, which is reproducing for the class $\Gamma_{a}$ minimizes the functional

$$
\|a(z) d z\|^{2}-2 \operatorname{Re} \int_{c} a d z
$$

over the class $\Gamma_{\alpha}$.

Proof. Let $a(z) d z$ be an element of $\Gamma_{\alpha}$. Then:

$$
\begin{aligned}
& \|a(z) d z-k(z, c) d z\|^{2} \\
& \quad=\|a(z) d z\|^{2}+\|k(z, c) d z\|^{2}-2 \operatorname{Re}(a(z) d z, k(z, c) d z) \\
& \quad=\|a(z) d z\|^{2}+\|k(z, c) d z\|^{2}-2 \operatorname{Re} \int_{c} a d z
\end{aligned}
$$

Therefore $\|a(z) d z\|^{2}-2 R e \int_{c} a d z=\|a(z) d z-k(z, c) d z\|^{2}-\|k(z, c)\|^{2}$ which proves the theorem. Notice that $\|k(z, c)\|^{2}=\int_{c} k(z, c) d z$, which shows that $\int_{c} k(z, c) d z$ is real.

2E. Let us now suppose that $\zeta_{1}$ and $\zeta_{2}$ are located in the same parametric disk. Consider the functions analytic on $W$ cut along $c$, except for the singularity $s=\log \left(z-\zeta_{1}\right) /\left(z-\zeta_{2}\right)$. The singularity for their differentials is $d z\left[1 /\left(z-\zeta_{1}\right)-1 /\left(z-\zeta_{2}\right)\right]$.

Assume that among these there exists a differential $d H_{c}$ which has the following property: for any $a d z \in \Gamma_{a e},\left(a d z, d H_{c}\right)=\int_{c} a d z$.

The differential $d H_{c}$ has an important extremal property.

THEOREM. The differential $d H_{c}$ minimizes the functional

$$
B(a)-R e \int_{c} a^{\prime} d z-d s
$$

among the class $\Gamma_{a e}(c)$ of differentials of functions a analytic on $W$ cut along c, except for the singularity

$$
s=\log \frac{z-\zeta_{1}}{z-\zeta_{2}}
$$

Proof. Let us first consider a compact bordered Riemann surface $\bar{W}$. Let $\alpha$ be a path around $c$, bounding a region $\Delta$. Let $W^{\prime}=\bar{W}-\Delta$. For any $d a=a^{\prime}(z) \in \Gamma_{a e}(c)$ one can write: 


$$
\begin{aligned}
& \left\|a^{\prime}(z) d z-d H_{c}\right\|_{W^{\prime}}^{2} \\
& \quad=\left\|a^{\prime}(z) d z\right\|_{W^{\prime}}^{2}-\left\|d H_{c}\right\|_{W^{\prime}}^{2}-2 R e\left(a^{\prime}(z) d z-d H_{c}, d H_{c}\right)_{W^{\prime}}
\end{aligned}
$$

Using the same method as in the proof of Theorem $2 \mathrm{C}$ this equality can be transformed into

$$
\begin{aligned}
\left\|a^{\prime}(z) d z-d H_{c}\right\|_{\bar{W}}^{2} & =2 B(a)-2 B\left(H_{c}\right)-2 \operatorname{Re}\left(a^{\prime}(z) d z-d H_{c}, d H_{c}\right)_{\bar{W}} \\
& =2 B(a)-2 B\left(H_{c}\right)-2 \operatorname{Re} \int_{c} a^{\prime}(z) d z-d H_{c}
\end{aligned}
$$

or

$$
\begin{aligned}
B(a)-\operatorname{Re} \int_{c} a^{\prime} d z-d s=B\left(H_{c}\right)-\operatorname{Re} \int_{c} d H_{c}-d s & \\
& +\frac{1}{2}\left\|a^{\prime}(z) d z-d H_{c}\right\|_{\bar{W}}^{2}
\end{aligned}
$$

which completes the proof.

To extend the property to open Riemann surfaces, we may use the method outlined in the proof of Theorem 2C. For canonical regions $\Omega$, the functional

$$
B_{\Omega}(\alpha)-\operatorname{Re} \int_{c} a^{\prime} d z-d s
$$

is easily seen to be nondecreasing when $\Omega$ increases. If there exists on $W$ cut along $c$ a function analytic except for the singularity $s$, then the functional has a finite limit and $H_{c \Omega}$ has a limit $H_{c}$ when $\Omega$ tends to $W$. By linearity one can extend the property to any finite chain.

\section{Chapter III. PLANAR RIEMANN SURFACES}

We shall now restrict our attention to planar Riemann surfaces $W$. We shall establish the connection between the principal functions for $W$ and the reproducing and orthogonal kernels, with our main interest devoted to the class $\Gamma_{a e}$.

\section{Reproducing and Orthogonal Kernels For $\Gamma_{a e}$.}

1A. In the case $\Gamma_{a}=\Gamma_{a e}$, we shall prove the following theorem:

Theorem. Let $W$ be a planar Riemann surface. If $P_{0}$ and $P_{1}$ denote the analytic principal functions on $W$ corresponding to the singularity $1 /(z-\zeta)$ and if $\left(\widetilde{k}_{0}(z, \zeta) / 2 \pi\right) d z$ and $\left(\widetilde{h}_{0}(z, \zeta) / 2 \pi\right) d z$ are respectively the reproducing and orthogonal (singularity $\left.(1 / 2 \pi)(z-\zeta)^{2}\right)$ kernels for $\Gamma_{a e}$ on $W$, then

$$
\frac{d}{d z} \frac{P_{1}-P_{0}}{2} d z=-\widetilde{k}_{0}(z, \zeta) d z
$$




$$
\frac{d}{d z} \frac{P_{1}+P_{0}}{2} d z=-\tilde{h}_{0}(z, \zeta) d z
$$

1B. First Proof. (By extremal property) Let $a(z) d z$ be an element of $\Gamma_{a e}$. Theorem 2B shows that

$$
\|a(z) d z\|^{2}-2 \operatorname{Rea}(\zeta)=\left\|a(z) d z-\frac{\widetilde{k}_{0}(z, \zeta)}{2 \pi} d z\right\|^{2}-\left\|\frac{\tilde{k}_{0}(z, \zeta)}{2 \pi} d z\right\|^{2}
$$

or

$$
\begin{aligned}
& \quad\|4 \pi a(z) d z\|^{2}-8 \pi \operatorname{Re}(4 \pi a(\zeta)) \\
& \quad=\left\|4 \pi a(z) d z-2 \widetilde{k}_{0}(z, \zeta) d z\right\|^{2}-\left\|2 \widetilde{k}_{0}(z, \zeta) d z\right\|^{2} .
\end{aligned}
$$

If we put $4 \pi a(z) d z=b(z) d z$, we get:

$$
\|b(z) d z\|^{2}-8 \pi \operatorname{Reb}(\zeta)=\left\|b(z) d z-2{\tilde{k_{0}}}_{(}(z, \zeta) d z\right\|^{2}-\left\|2 \widetilde{k}_{0}(z, \zeta) d z\right\|^{2} .
$$

But the analytic function $P_{0}-P_{1}=Q$ corresponding to the singularity $1 /(z-\zeta)$ minimize the expression

$$
D(U)-\left.4 \pi R e \frac{d U}{d z}\right|_{z=\zeta}=D(U-Q)-D(Q)
$$

among all analytic functions on $W$. In terms of the differential $d U$, the expression becomes:

$$
\frac{1}{2}\|d U\|^{2}-\left.4 \pi R e \frac{d U}{d z}\right|_{z=\zeta}=\frac{1}{2}\|d U-d Q\|^{2}-\frac{1}{2}\|d Q\|^{2}
$$

or

$$
\|d U\|^{2}-\left.8 \pi R e \frac{d U}{d z}\right|_{z=\zeta}=\|d U-d Q\|^{2}-\|d Q\|^{2}
$$

which shows that $(d Q / d z) d z$ minimizes the same functional as $2 k_{0}(z, \zeta) d z$ over $\Gamma_{a e}$. Therefore

$$
\frac{d}{d z} \frac{\left(P_{1}-P_{0}\right)}{2} d z=-\widetilde{k}_{0}(z, \zeta) d z
$$

An analogous proof will show,

$$
\frac{d}{d z} \frac{P_{1}+P_{0}}{2} d z=-\widetilde{h}_{0}(z, \zeta) d z
$$

1C. Second Proof. (By canonical exhaustion) 
Let $\bar{W}$ be a planar compact bordered Riemann surface, with border $\beta$. We shall first prove a lemma on the boundary behavior of the differentials of $P_{0}-P_{1}$ and $P_{0}+P_{1}$.

Lemma. Along $\beta$

$$
\overline{\frac{d\left(P_{0}-P_{1}\right)}{d z} d z}=\frac{d\left(P_{0}+P_{1}\right)}{d z} d z .
$$

Proof.

$$
\begin{aligned}
& \frac{1}{2} \frac{d P_{0}}{d z}=\frac{\partial p_{0}}{d x}-i \frac{\partial p_{0}}{\partial y} \\
& \frac{1}{2} \frac{d P_{1}}{d z}=\frac{\partial p_{1}}{\partial x}-i \frac{\partial p_{1}}{\partial y} .
\end{aligned}
$$

For a special choice of the local variable $d z=d x$ along $\beta$ and then by definition

$$
\begin{aligned}
& \frac{\partial p_{0}}{\partial y}=0 \\
& \frac{\partial p_{1}}{\partial x}=0 .
\end{aligned}
$$

Therefore the equality in the lemma corresponds to

$$
\overline{\left(\frac{\partial p_{0}}{\partial x}+i \frac{\partial p_{1}}{\partial y}\right) d x}=\left(\frac{\partial p_{0}}{\partial x}-i \frac{\partial p_{1}}{\partial x}\right) d x .
$$

We may notice that the proof actually shows that for any compact bordered $\bar{W}$

$$
\left(\frac{\partial p_{0}}{\partial z}-i \frac{\partial p_{1}}{\partial z}\right) d z=\overline{\left(\frac{\partial p_{0}}{\partial z}+i \frac{\partial p_{1}}{\partial z}\right) d z} \quad \text { along } \beta
$$

We shall now show directly that

$$
-\frac{1}{2 \pi} \frac{d}{d z} \frac{\left(P_{1}-P_{0}\right)}{2} d z
$$

is the reproducing kernel for $\Gamma_{a e}$ on $\bar{W}$. Let $d a=a^{\prime}(z) d z$ be an element of $\Gamma_{a e}$, then

$$
\left(a^{\prime}(z) d z, \frac{d}{d z} \frac{\left(P_{1}-P_{0}\right)}{2} d z\right)=-\frac{1}{i} \int_{\bar{w}} a^{\prime}(z) \frac{\bar{d} \frac{\left(P_{1}-P_{0}\right)}{d z}}{2} d z \overline{d z} .
$$

But we know that

$$
\int_{\bar{W}} \omega d f=\int_{\beta} \omega f-\int_{\bar{W}} f d \omega
$$


If $\omega$ is closed, then

$$
\int_{\bar{W}} \omega d f=\int_{\beta} \omega f
$$

Therefore:

$$
\begin{aligned}
\left(a^{\prime}(z) d z, \frac{d}{d z} \frac{\left(P_{1}-P_{0}\right)}{2} d z\right) & =-\frac{1}{i} \int_{\beta} a(z) \frac{d}{d z} \frac{\overline{\left(P_{1}-P_{0}\right)}}{2} \overline{d z} \\
& =\frac{1}{i} \int_{\beta}(\alpha z) \frac{d}{d z} \frac{\left(P_{1}+P_{0}\right)}{2} d z
\end{aligned}
$$

by the property proved in the lemma. Let $\alpha$ be the boundary of a circle of radius $r$ about $z=\zeta, r$ being small enough for $\alpha$ to lie in the same parametric disk as $z=\zeta$; then:

$$
\frac{1}{i} \int_{\beta} a(z) \frac{d}{d z} \frac{\left(P_{1}+P_{0}\right)}{2} d z=\frac{1}{i} \int_{a} a(z) \frac{d}{d z} \frac{\left(P_{1}+P_{0}\right)}{2} d z
$$

In a neighborhood of $z=\zeta,\left(P_{1}+P_{0}\right) / 2$ may be expressed as:

$$
\frac{P_{1}+P_{0}}{2}=\frac{1}{z-\zeta}+l(z, \zeta)
$$

where $l(z, \zeta)$ is analytic in $z$. Therefore

$$
\frac{1}{i} \int_{a} a(z) \frac{d}{d z} \frac{P_{1}+P_{0}}{2} d z=-\frac{1}{i} \int_{a} \frac{a(z)}{(z-\zeta)^{2}} d z+\frac{1}{i} \int_{a} a(z) \frac{d l(z, \zeta)}{d z} d z
$$

The second integral tends to zero as $r$ tends to zero, for the integrand is analytic at $\zeta$. The first one has the value $-2 \pi a^{\prime}(\zeta)$. We conclude that

$$
\left(a^{\prime}(z) d z,-\frac{1}{2 \pi} \frac{d}{d z} \frac{\left(P_{1}-P_{0}\right)}{2} d z\right)=a^{\prime}(\zeta)
$$

which proves the reproducing property.

The same method can be used to prove that $(-1 / 2 \pi)(d / d z)\left(\left(P_{1}+P_{0}\right) /\right.$ $2) d z$ is the orthogonal kernel for $\Gamma_{a e}$, corresponding to the singularity $d z / 2 \pi(z-\zeta)^{2}$. For $a^{\prime}(z) d z \in \Gamma_{a e}$, we compute the following inner product:

$$
\begin{aligned}
\left(a^{\prime}(z) d z, \frac{d}{d z} \frac{\left(P_{1}+P_{0}\right)}{2} d z\right) & =-\frac{1}{i} \int_{\bar{W}} a^{\prime}(z) \frac{\bar{d} \frac{\left(P_{1}+P_{0}\right)}{d z}}{2} d z \overline{d z} \\
& =-\frac{1}{i} \int_{\beta} a(z) \frac{d}{\frac{d\left(P_{1}+P_{0}\right)}{d z} d z}
\end{aligned}
$$




$$
\begin{aligned}
& =-\frac{1}{i} \int_{\beta} a(z) \frac{d}{d z} \frac{\left(P_{1}-P_{0}\right)}{2} d z \\
& =-\frac{1}{i} \int_{\alpha} a(z) \frac{d}{d z} \frac{\left(P_{1}-P_{0}\right)}{2} d z .
\end{aligned}
$$

The last integral tends to zero when $r$ tends to zero for the integrand is analytic at $z=\zeta$. Therefore

$$
\left(a^{\prime}(z) d z,-\frac{1}{2 \pi} \frac{d}{d z} \frac{\left(P_{1}+P_{0}\right)}{2} d z\right)=0 .
$$

1D. To extend the property to open Rieman surfaces $W$, we shall consider a canonical exhaustion of $W$ by regular regions $\Omega$

Let $d a=a^{\prime}(z) d z$ be an exact analytic differential on $W$; we denote by

$P_{i \Omega} i=(0,1)$ the analytic principal functions for the region $\Omega$ and by

$P_{i} \quad i=(0,1)$ the analytic principal functions corresponding to the surface $W$. For each $\Omega \subset W$, we proved that:

$$
\left(a^{\prime}(z) d z,-\frac{1}{2 \pi} \frac{d}{d z} \frac{\left(P_{1 \Omega}-P_{0 \Omega \Omega}\right)}{2} d z\right)_{\Omega}=a^{\prime}(\zeta)
$$

We now consider:

$$
\begin{aligned}
& \left(a^{\prime}(z) d z,-\frac{1}{2 \pi} \frac{d}{d z} \frac{\left(P_{1}-P_{0}\right)}{2} d z\right)_{W}-a^{\prime}(\zeta) \\
& =\left(a^{\prime}(z) d z,-\frac{1}{2 \pi} \frac{d}{d z} \frac{\left(P_{1}-P_{0}\right)}{2} d z\right)_{W}-\left(a^{\prime}(z) d z,-\frac{1}{2 \pi} \frac{d}{d z} \frac{\left(P_{1 \Omega}-P_{0 \Omega \Omega}\right)}{2} d z\right)_{\Omega} \\
& =\left(a^{\prime}(z) d z,-\frac{1}{2 \pi} \frac{d}{d z} \frac{\left[\left(P_{1}-P_{1 \Omega}\right)-\left(P_{0}-P_{0 \Omega}\right)\right]}{2} d z\right)_{\Omega} \\
& +\left(a^{\prime}(z) d z,-\frac{1}{2 \pi} \frac{d}{d z} \frac{\left(P_{1}-P_{0}\right)}{2} d z\right)_{W-\Omega} .
\end{aligned}
$$

Therefore:

$$
\begin{aligned}
& \left|\left(a^{\prime}(z) d z,-\frac{1}{2 \pi} \frac{d}{d z} \frac{\left(P_{1}-P_{0}\right)}{2} d z\right)_{W}-a^{\prime}(\zeta)\right| \\
& \leqq\left|\left(a^{\prime}(z) d z,-\frac{1}{2 \pi} \frac{d}{d z} \frac{\left[\left(P_{1}-P_{1 \Omega}\right)-\left(P_{0}-P_{0 \Omega}\right)\right]}{2} d z\right)_{\Omega}\right| \\
& +\left|\left(a^{\prime}(z) d z,-\frac{1}{2 \pi} \frac{d}{d z} \frac{\left(P_{1}-P_{0}\right)}{2} d z\right)_{W-\Omega}\right| .
\end{aligned}
$$

By Schwarz's inequality: 


$$
\begin{aligned}
& \left|\left(a^{\prime}(z) d z,-\frac{1}{2 \pi} \frac{d}{d z} \frac{\left(P_{1}-P_{0}\right)}{2} d z\right)_{W-\Omega}\right| \\
& \quad \leqq\left\|a^{\prime}(z) d z\right\|_{W-\Omega}\left\|-\frac{1}{2 \pi} \frac{d}{d z} \frac{\left(P_{1}-P_{0}\right)}{2} d z\right\|_{W-\Omega}
\end{aligned}
$$

both norms in the right hand side tend to zero when $\Omega \rightarrow W$ because $a^{\prime}(z) d z$ has finite norm and

$$
\left\|-\frac{1}{2 \pi} \frac{d}{d z} \frac{\left(P_{1}-P_{0}\right)}{2} d z\right\|_{W-\Omega}=\sqrt{2} D_{W-\Omega}\left[\frac{p_{1}-p_{0}}{4 \pi}\right]^{1 / 2}
$$

and $p_{1}-p_{0}$ has finite Dirichlet integral.

As to the first expression in the right hand side of the inequality:

$$
\begin{aligned}
& \left|\left(a^{\prime}(z) d z,-\frac{1}{2 \pi} \frac{d}{d z} \frac{\left[\left(P_{1}-P_{1 \Omega}\right)-\left(P_{0}-P_{0 \Omega}\right)\right]}{2} d z\right)_{\Omega}\right| \\
& \quad \leqq\left\|a^{\prime}(z) d z\right\|_{\Omega}\left\|-\frac{1}{2 \pi} \frac{d}{d z} \frac{\left[\left(P_{1}-P_{1 \Omega}\right)-\left(P_{0}-P_{0 \Omega}\right)\right]}{2} d z\right\|_{\Omega} ;
\end{aligned}
$$

$\left\|a^{\prime}(z) d z\right\|_{\Omega}$ has a finite limit when $\Omega \rightarrow W$ and

$$
\left\|-\frac{1}{2 \pi} \frac{d}{d z} \frac{\left[\left(P_{1}-P_{1 \Omega}\right)-\left(P_{0}-P_{0 \Omega}\right)\right]}{2} d z\right\|_{\Omega}=2 D_{\Omega}^{1 / 2} \frac{1}{4 \pi}\left[\left(p_{1}-p_{1 \Omega}\right)-\left(p_{0}-p_{0 . \Omega}\right)\right] .
$$

Moreover by the triangle inequality

$$
\begin{aligned}
D_{\Omega}^{1 / 2} & \left(\frac{1}{4 \pi}\left[\left(p_{1}-p_{1 \Omega}\right)-\left(p_{0}-p_{0 \Omega}\right)\right]\right) \\
& \leqq D_{\Omega}^{1 / 2}\left(\frac{1}{4 \pi}\left(p_{1}-p_{1 \Omega}\right)\right)+D_{\Omega}^{1 / 2}\left(\frac{1}{4 \pi}\left(p_{0}-p_{0 \Omega}\right)\right) .
\end{aligned}
$$

But Theorem $1 \mathrm{~F}$, Ch. I shows that

$$
\lim _{\Omega \rightarrow W} D_{\Omega}\left(p_{i}-p_{i \Omega}\right)=0 \quad i=0,1 .
$$

We conclude that

$$
\left(a^{\prime}(z) d z,-\frac{1}{2 \pi} \frac{d}{d z} \frac{\left(P_{1}-P_{0}\right)}{2} d z\right)_{W}=a^{\prime}(\zeta) .
$$

The same pattern of proof applies to show that

$$
\left(a^{\prime}(z) d z,-\frac{1}{2 \pi} \frac{d}{d z} \frac{\left(P_{1}+P_{0}\right)}{2} d z\right)_{W}=0
$$

which proves that $(-1 / 2 \pi)(d / d z)\left(\left(P_{1}+P_{0}\right) / 2\right) d z$ is the kernel orthogonal 
to $\Gamma_{a e}$ corresponding to the singularity $1 / 2 \pi(z-\zeta)^{2}$.

By the uniqueness property we have proved that:

$$
\begin{aligned}
& \frac{d}{d z} \frac{\left(P_{1}-P_{0}\right)}{2} d z=-\widetilde{k}_{0}(z, \zeta) d z \\
& \frac{d}{d z} \frac{\left(P_{1}+P_{0}\right)}{2} d z=-\widetilde{h}_{0}(z, \zeta) d z .
\end{aligned}
$$

1E. We now investigate the relationship between the principal functions corresponding to the singularity $\left(1 /(z-\zeta)^{m+1}\right) m>0$ and the kernels $\widetilde{k}_{m}(z, \zeta) d z$ and $\widetilde{h}_{m}(z, \zeta) d z$ (singularity $\left.\left(1 /(z-\zeta)^{m+2}\right) m>0\right)$. In the statement of the next theorem we call $P_{0 m}$ and $P_{1 m}$ the analytic principal functions corresponding to the singularity $1 /(z-\zeta)^{m+1}$.

THEOREM.

$$
\begin{aligned}
& \frac{1}{m+1} \frac{d}{d z} \frac{\left(P_{1 m}-P_{0 m}\right)}{2} d z=-\widetilde{k}_{m}(z, \zeta) d z \\
& \frac{1}{m+1} \frac{d}{d z} \frac{\left(P_{1 m}+P_{0 m}\right)}{2} d z=-\widetilde{h}_{m}(z, \zeta) d z .
\end{aligned}
$$

Proof. We shall prove that both sides in the first equation minimize the same functional over the class $\Gamma_{a e}$. We know by Theorem $2 \mathrm{~B}$, Ch. II that

$$
\frac{(m+1) !}{2 \pi} \widetilde{k}_{m}(z, \zeta) d z
$$

minimizes

$$
\|a(z) d z\|^{2}-2 \operatorname{Re} a^{(m)}(\zeta) \text { over } \Gamma_{a e},
$$

and by Theorem 1E, Ch. I that

$$
Q_{m}=P_{0 m}-P_{1 m}
$$

minimizes

$$
D(U)-\left.\frac{4 \pi}{m !} R \mathrm{e} \frac{d^{m+1} U}{d z^{m+1}}\right|_{z=\zeta}
$$

The last expression can be written as:

$$
2\|d U\|^{2}-\left.\frac{2 \pi}{m !} R e \frac{d^{m}}{d z^{m}}\left(\frac{d U}{d z}\right)\right|_{z=\zeta}
$$


Therefore $(m ! / 2 \pi)\left(d Q_{m} / d z\right) d z$ minimizes the same expression as $((m+1) ! /$ $2 \pi) \widetilde{k}_{m}(z, \zeta) d z$ over $\Gamma_{a e}$. Then

$$
\frac{1}{m+1} \frac{d}{d z} \frac{\left(P_{1 m}-P_{0 m}\right)}{2} d z=-\widetilde{k}_{m}(z, \zeta) d z .
$$

To prove the second equality, one can show by the method outlined in the second proof of Theorem $1 \mathrm{~A}$ that

$$
\frac{1}{m+1} \frac{d}{d z} \frac{\left(P_{1 m}+P_{0 m}\right)}{2} d z
$$

is the orthogonal kernel for $\Gamma_{a e}$ corresponding to the singularity $d z /(z-\zeta)^{m+2}, m>0$; then by the uniqueness theorem it follows that:

$$
\frac{1}{m+1} \frac{d}{d z} \frac{\left(P_{1 m}+P_{0 m}\right)}{2} d z=-\widetilde{h}_{m}(z, \zeta) d z .
$$

1F. We now may use our knowledge about $\widetilde{k}_{m}(z, \zeta) d z$ and $\widetilde{h}_{m}(z, \zeta) d z$ to obtain new results about the functions $P_{0 m}$ and $P_{1 m}$.

THEOREM. Between the derivatives of $P_{i m}$ and $P_{i}, i=0,1$, the following relations hold.

$$
\begin{aligned}
& \frac{d}{d z}\left(P_{1 m}+P_{0 m}\right)=\frac{1}{m !} \frac{d}{d z} \frac{d^{m}}{d z^{m}}\left(P_{1}+P_{0}\right) \\
& \frac{d}{d \bar{z}} \overline{\left(P_{1 m}-P_{0 m}\right)}=\frac{1}{m !} \frac{d}{d \bar{z}} \frac{d^{m}}{d \zeta^{m}} \overline{\left(P_{1}-P_{0}\right)}
\end{aligned}
$$

moreover:

$$
\begin{aligned}
& \frac{d}{d z}\left(P_{1}+P_{0}\right) \quad \text { is symmetric in } z \text { and } \zeta \\
& \frac{d}{d z}\left(P_{1}-P_{0}\right) \text { is conjugate symmetric in } z \text { and } \zeta .
\end{aligned}
$$

Proof. From the relations

$$
\begin{aligned}
& \widetilde{\widetilde{k}_{m}(z, \zeta)}=\frac{1}{(m+1) !} \frac{d^{m}}{d \zeta^{m}} \overline{\widetilde{k}_{0}(z, \zeta)} \\
& \widetilde{h}_{m}(z, \zeta)=\frac{1}{(m+1) !} \frac{d^{m}}{d z^{m}} \widetilde{h}_{0}(z, \zeta),
\end{aligned}
$$

it follows that

$$
\frac{1}{m+1} \frac{d}{d \bar{z}} \overline{\left(P_{1 m}-P_{0 m}\right)}=\frac{1}{(m+1) !} \frac{d}{d \bar{z}} \frac{d^{m}}{d \zeta^{m}} \overline{\left(P_{1}-P_{0}\right)}
$$


or

$$
\frac{d}{d \bar{z}} \overline{\left(P_{1 m}-P_{0 m}\right)}=\frac{1}{m !} \frac{d}{d \bar{z}} \frac{d^{m}}{d \xi^{m}} \overline{\left(P_{1}-P_{0}\right)}
$$

Similarly

$$
\frac{d}{d z}\left(P_{1 m}+P_{0 m}\right)=\frac{1}{m !} \frac{d^{m}}{d z^{m}}\left(P_{1}+P_{0}\right)
$$

and one gets the first set of relations in the theorem. To get the second set, we recall that:

$$
\begin{aligned}
& \tilde{h}_{0}(z, \zeta)=\widetilde{h}_{0}(\zeta, z) \\
& \tilde{k}_{0}(z, \zeta)=\widetilde{\tilde{k}_{0}(\zeta, z)}
\end{aligned}
$$

It follows that

$$
\frac{d}{d z}\left(P_{1}+P_{0}\right) \text { is symmetric in } z \text { and } \zeta
$$

and

$$
\frac{d}{d z}\left(P_{1}-P_{0}\right) \text { is conjugate symmetric in } z \text { and } \zeta
$$

1G. To complete our study, we shall consider the case of the singularity $\left(1 /\left(z-\zeta_{1}\right)-1 /\left(z-\zeta_{2}\right)\right) d z$, where $\zeta_{1}$ and $\zeta_{2}$ lie in the same parametric disk.

Let $P_{c 0}$ and $P_{c 1}$ be the principal functions corresponding to the singularity $\log \left(z-\zeta_{1}\right) /\left(z-\zeta_{2}\right) . \quad P_{c 0}$ and $P_{c 1}$ are analytic on $W$ cut along a path $c$ joining $\zeta_{1}$ to $\zeta_{2}$. We shall prove the following results:

1H. THEOREM. The derivatives of $P_{c 0}, P_{c 1}$ and the kernels $\tilde{k}(z, c) d z$, $\tilde{h}(z, c) d z$ are connected by the relations:

$$
\begin{aligned}
& \frac{d}{d z}\left(\frac{P_{c 1}-P_{c 0}}{2}\right) d z=\tilde{k}(z, c) d z \\
& \frac{d}{d z}\left(\frac{P_{c 1}+P_{c 0}}{2}\right) d z=\tilde{h}(z, c) d z .
\end{aligned}
$$

Proof. $\quad(\widetilde{k}(z, c) / 2 \pi) d z$ is known to minimize the functional

$$
\left\|a^{\prime}(z) d z\right\|^{2}+2 \operatorname{Re}\left(a\left(\zeta_{2}\right)-a\left(\zeta_{1}\right)\right)
$$

among all $d a=a^{\prime}(z) d z$ in $\Gamma_{a e}$.

But $P_{c 0}-P_{c 1}=Q_{c}$ minimizes

$$
D(U)+4 \pi \operatorname{Re}\left(U\left(\zeta_{1}\right)-U\left(\zeta_{2}\right)\right) \text { over the class of analytic }
$$


functions $U$ on $W$.

The last expression can be written as:

$$
\frac{1}{2}\left[\|d U\|^{2}-8 \pi R \mathrm{e}\left(U\left(\zeta_{2}\right)-U\left(\zeta_{1}\right)\right] .\right.
$$

By comparison of the two functionals it follows that

$$
\frac{d}{d z}\left(\frac{P_{c 1}-P_{c 0}}{2}\right) d z=\tilde{k}(z, c) d z
$$

To get the second relation mentioned in the theorem, one can either follow the method outlined in Theorem 1C, Ch III, second proof, where $\alpha$ is now a path around the cut $c$ in some parametric disk containing $\zeta_{1}$ and $\zeta_{2}$, or notice that $\tilde{h}(z, c) d z$ and $(d / d z)\left(\left(P_{c}+P_{c 0}\right) / 2\right) d z$ minimize the functional:

$$
B(a)-R e \int_{c} a^{\prime} d z-d s
$$

over the class $\Gamma_{a e}(c)$ of differential of functions $a$, analytic on $W$ cut along $c$, expect for the singularity $s=\log \left(\left(z-\zeta_{1}\right) /\left(z-\zeta_{2}\right)\right)$.

1I. We prove next a theorem establishing a relation between the derivatives of $P_{c 0}$ and $P_{c 1}$, and the principal functions $P_{0}$ and $P_{1}$.

THEOREM. Let $P_{c 0}$ and $P_{c 1}$ be the principal functions on $W$ cut along $c$ corresponding to the singularity $\log \left(\left(z-\zeta_{1}\right) /\left(z-\zeta_{2}\right)\right)$. If we denote by $P_{0}(z, \zeta), P_{1}(z, \zeta)$ the principal functions on $W$ corresponding to the singularity $1 /(z-\zeta)$ with $P_{0}=p_{0}+i p_{0}^{*}, P_{1}=p_{1}+i p_{1}^{*}$ then

$$
\begin{aligned}
\frac{d}{d z} P_{c 1} & =p_{1}\left(\zeta_{2}, z\right)-p_{1}\left(\zeta_{1}, z\right)+i\left(p_{0}^{*}\left(\zeta_{2}, z\right)-p_{0}^{*}\left(\zeta_{1}, z\right)\right) \\
\frac{d}{d z} P_{c 0} & =i\left(p_{1}^{*}\left(\zeta_{2}, z\right)-p_{1}^{*}\left(\zeta_{1}, z\right)\right)+p_{0}\left(\zeta_{2}, z\right)-p_{0}\left(\zeta_{1}, z\right) .
\end{aligned}
$$

Proof. We recall the equalities:

$$
\begin{aligned}
& \widetilde{k}(z, c)=\int_{c} \widetilde{k}_{0}(z, \zeta) \overline{d \zeta} \\
& \widetilde{h}(z, c)=\int_{c} \widetilde{h}_{0}(z, \zeta) d \zeta,
\end{aligned}
$$

and the symmetry relations:

$$
\widetilde{k}_{0}(z, \zeta)=\overline{\widetilde{k}_{0}(\zeta, z)} \quad \tilde{h}_{0}(z, \zeta)=\tilde{h}_{0}(\zeta, z) .
$$

Therefore: 


$$
\begin{aligned}
\frac{d}{d z}\left(P_{c 1}-P_{c 0}\right) & =\int_{c} \frac{d}{d z}\left[P_{1}(z, \zeta)-P_{0}(z, \zeta)\right] \overline{d \zeta} \\
& =\int_{c} \frac{d}{d \zeta}\left[P_{1}(\zeta, z)-P_{0}(\zeta, z)\right] d \zeta \\
& =P_{1}\left(\zeta_{2}, z\right)-P_{1}\left(\zeta_{1} z\right)-P_{0}\left(\zeta_{2}, z\right)+P_{0}\left(\zeta_{1}, z\right) \\
\frac{d}{d z}\left(P_{c 1}+P_{c 0}\right) & =\int_{c} \frac{d}{d z}\left[P_{1}(z, \zeta)+P_{0}(z, \zeta)\right] d \zeta \\
& =\int_{c} \frac{d}{d \zeta}\left[P_{1}(\zeta, z)+P_{0}(\zeta, z)\right] d \zeta \\
& =P_{1}\left(\zeta_{2}, z\right)-P_{1}\left(\zeta_{1}, z\right)+P_{0}\left(\zeta_{2}, z\right)-P_{0}\left(\zeta_{1}, z\right)
\end{aligned}
$$

by addition and subtraction we obtain

$$
\begin{aligned}
& \frac{d P_{c 1}}{d z}=p_{1}\left(\zeta_{2}, z\right)-p_{1}\left(\zeta_{1}, z\right)+i p_{0}^{*}\left(\zeta_{2}, z\right)-i p_{0}^{*}\left(\zeta_{1}, z\right) \\
& \frac{d P_{c 0}}{d z}=i p_{1}^{*}\left(\zeta_{2}, z\right)-i p_{1}^{*}\left(\zeta_{1}, z\right)+p_{0}\left(\zeta_{2}, z\right)-p_{0}\left(\zeta_{1}, z\right) .
\end{aligned}
$$

\section{Reproducing and Orthogonal Kernels for $\Gamma_{a}$.}

2A. We shall now again restrict ourselves to the compact bordered case, and investigate the relationship between the principal functions and Green's and Neumann functions [Schiffer [5], Bergman [2]).

Let $\bar{W}$ be a planar compact bordered Riemann surface with $k$ contours $\beta_{i}, i=1 \cdots k$. We recall the definition of the Green's function $g(z, \zeta)$ for $\bar{W}$.

Definition. The Green's function $g(z, \zeta)$ for $\bar{W}$ is defined in the following way:

(a) $g(z, \zeta)$ is harmonic on $\bar{W}$ except at $z=\zeta$

(b) $g(z, \zeta)+\log |z-\zeta|$ is harmonic at $z=\zeta$

(c) $g(z, \zeta)=0$ on $\beta=\bigcup_{i=1}^{k} \beta_{i}$.

The function $g(z, \zeta)$ possesses a harmonic conjugate $g^{*}(z, \zeta)$ and one may construct the function

$$
G(z, \zeta)=g+i g^{*}
$$

$G$ is harmonic in $\zeta$, analytic in $z$; it has a logarithmic pole with coefficient 1 at $\zeta$ and is determined up to an additive constant. It is not single valued. 
2B. We introduce the harmonic measure of a boundary component $\beta_{i}$.

Definition. The harmonic measure of the boundary component $\beta_{i}$ with respect to $W$ is the harmonic function $\omega_{i}(z)$ taking the following boundary values:

$$
\begin{array}{lll}
\omega_{i}(z)=0 & \text { on } \beta_{j} \quad j \neq i \\
\omega_{i}(z)=1 & \text { on } \beta_{i} .
\end{array}
$$

The period of $G(z, \zeta)$ along $\beta_{i}$ is easily seen to be $2 \pi i \omega_{i}(\zeta)$.

We may associate to the harmonic measure an analytic function:

$$
w_{i}(z)=\omega_{i}(z)+i \omega_{i}^{*}(z) .
$$

The period of $w_{i}(z)$ around $\beta_{j}$ will be denoted by $-2 \pi i P_{j i}$. It is easy to show that:

1. $P_{i j}=P_{j i}$.

2. The $k \times k$ matrix $\left(P_{i j}\right)$ is positive semi-definite.

3. The $(k-1) \times(k-1)$ matrix $\left(P_{i j}\right)_{1,2 \ldots k-1}$ is positive definite.

2C. Let us now introduce the function

$$
F(z, \zeta)=\exp (-G(z, \zeta))
$$

$F(z, \zeta)$ is not in general single-valued. To get a single valued function we shall add to $G(z, \zeta)$ an appropriate linear combination of functions $w_{i}(z)$; to do so, we solve the system:

$$
\sum_{j=1}^{k-1} P_{i j} u_{j}(\zeta)=\omega_{i}(\zeta) \quad(i=1 \cdots k-1)
$$

in the form

$$
u_{j}(\zeta)=\sum_{i=1}^{k-1} \Pi_{j i} \omega_{i}(\zeta) \quad \text { with } \quad\left(\Pi_{j i}\right)_{1 \ldots k-1}=\left(P_{i j}\right)_{1 \cdots k-1}^{-1} .
$$

The function $w(z, \zeta)=\sum_{j=1}^{k-1} u_{j}(\zeta) w_{j}(z)$ is analytic in $z$ and has period $-2 \pi i \sum_{j=1}^{k-1} P_{i j} u_{j}(\zeta)=-2 \pi i \omega_{i}(\zeta)$ along $\beta_{i}$.

Therefore

$$
\log f(z, \zeta)=-\left[G(z, \zeta)+\sum_{j=1}^{k-1} u_{j}(\zeta) w_{j}(z)\right]
$$

has period zero along $\beta_{i}, i=1 \cdots k-1$. Its period along $\beta_{k}$ is $2 \pi i$. 
Therefore

$$
f(z, \zeta)=\exp \left\{-\left[G(z, \zeta)+\sum_{i=1}^{k-1} \sum_{j=1}^{k-1} \Pi_{j i} w_{i}(\zeta) w_{j}(z)\right]\right\}
$$

is single valued on $\bar{W}$. It has a simple zero at $z=\zeta$, and from the known boundary behavior of $g(z, \zeta)$ and $w_{j}(z)$,

$$
\log |f(z, \zeta)|=\operatorname{Re}\{\log f(z, \zeta)\}= \begin{cases}0 & \text { on } \beta_{k} \\ -u_{i}(\zeta) & \text { on } \beta_{i}, i<k\end{cases}
$$

It follows, by differentiation with respect to $\zeta(\zeta=\xi+i \eta)$ that $\operatorname{Re}(\partial / \partial \xi) \log f(z, \zeta)=\lambda_{i}(\zeta)$ on $\beta_{i}$, where $\lambda_{i}(\zeta)$ is a constant and that $\Psi(z, \zeta)=-(\partial / \partial \xi) \log f(z, \zeta)$ has a constant real part on each $\beta_{i}$. Moreover, it has at $z=\zeta$ a simple pole with residue 1 . Except for an additive constant, it coincides then with the principal function $P_{1}$. In the same way $\Phi(z, \zeta)=-(1 / i)(\partial / \partial \eta) \log f(z, \zeta)$ has a constant imaginary part on each $\beta_{i}$. At $z=\zeta$ it has a single pole with residue 1 . Except for an additive constant it coincides then with the principal function $P_{0}$.

2D. We now define the Neumann function $N(z, \zeta)$ by the following properties:

(a) $N(z, \zeta)$ is harmonic for $z \in \bar{W}$ except at the point $z=\zeta$

(b) $N(z, \zeta)+\log |z-\zeta|$ is harmonic at $z=\zeta$

(c) $(\partial / \partial n) N(z, \zeta)=(2 \pi / L)$ for $z \in \beta$ where $L$ is the total length of $\beta$ $N$ is defined up to an additive constant depending on $\zeta$. To fix $N$ completely we require that:

(d) $\int_{\beta} N d z=0$.

Between the derivatives of $N$ and $g$ the following relations hold:

$$
\begin{aligned}
& \frac{\partial^{2} N(z, \zeta)}{\partial z \partial \zeta}=\frac{\partial^{2} g(z, \zeta)}{\partial z \partial \zeta}+\frac{1}{4} \sum_{i=1}^{k-1} \sum_{j=1}^{k-1} \Pi_{i j} w_{i}^{\prime}(z) w_{j}^{\prime}(\zeta) \\
& \frac{\partial^{2} N(z, \zeta)}{\partial z \overline{\partial \zeta}}=-\frac{\partial^{2} g(z, \zeta)}{\partial z \overline{\partial \zeta}}-\frac{1}{4} \sum_{i=1}^{k-1} \sum_{j=1}^{k-1} \Pi_{i j} w_{i}^{\prime}(z) \overline{w_{j}^{\prime}(\zeta)}
\end{aligned}
$$

We now fix our attention on the function

$$
m(z, \zeta)=-\log |f(z, \zeta)|=-\frac{1}{2} \log f(z, \zeta)-\frac{1}{2} \log \overline{f(z, \zeta)}
$$

which is harmonic in $z$ and constant on each $\beta_{i}$. It has a logarithmic pole with coefficient -1 .

It is a consequence of the definition that

$$
m(z, \zeta)=g(z, \zeta)+\sum_{i=1}^{k-1} \sum_{j=1}^{k-1} \Pi_{i j} w_{i}(z) w_{j}(\zeta)
$$

Therefore: 


$$
\begin{aligned}
& \frac{\partial^{2} N}{\partial z \partial \zeta}=\frac{\partial^{2} m}{\partial z \partial \zeta} \\
& \frac{\partial^{2} N}{\partial z \overline{\partial \zeta}}=\frac{\partial^{2} m}{\partial z \overline{\partial \zeta}}
\end{aligned}
$$

2E. We shall consider reproducing and orthogonal kernels for the space $\Gamma_{a}$ on $\bar{W}$. In terms of the inner product we used for $\Gamma_{a}$, the expressions for the Bergman Kernel corresponding to $\Gamma_{a}$ are

$$
\begin{aligned}
& K(z, \bar{\zeta}) d z=-\frac{1}{\pi} \frac{\partial^{2} g(z, \zeta)}{\partial z \overline{\partial \zeta}} d z \\
& L(z, \zeta) d z=-\frac{1}{\pi} \frac{\partial^{2} g(z, \zeta)}{\partial z \partial \zeta} d z
\end{aligned}
$$

From the properties of the Green's function it can easily be derived that for $a(z) d z \in \Gamma_{a}$

$$
\begin{aligned}
& (a(z) d z, K(z, \bar{\zeta}) d z)=a(\zeta) \\
& (a(z) d z, L(z, \zeta) d z)=0
\end{aligned}
$$

$K(z, \bar{\zeta}) d z$ is then the reproducing kernel for $\Gamma_{a}, L(z, \zeta) d z$ is then the orthogonal kernel for $\Gamma_{a}$ corresponding to the singularity $d z / 2 \pi(z-\zeta)^{2}$. It is known that

$$
L(z, \zeta)=L(\zeta, z), \quad K(z, \bar{\zeta})=\overline{K(\zeta, \bar{z})}
$$

and for $z \in \beta, \zeta \in \bar{W}$

$$
L(z, \zeta) d z=-\overline{K(z, \bar{\zeta}) d z}
$$

2E. For $\Gamma_{a e}$ one defines in a similar way

$$
\begin{aligned}
& K_{0}(z, \bar{\zeta}) d z=\frac{1}{\pi} \frac{\partial^{2} N(z, \zeta)}{\partial z \partial \bar{\zeta}} \\
& L_{0}(z, \zeta) d z=-\frac{1}{\pi} \frac{\partial^{2} N(z, \zeta)}{\partial z \partial \zeta}
\end{aligned}
$$

which, from the properties of the Neumann functions can be shown to be the corresponding reproducing and orthogonal kernels.

We recall that we had, up to additive constants,

$$
\begin{aligned}
& P_{1}=-\frac{\partial}{\partial \xi} \log f(z, \zeta) \\
& P_{0}=-\frac{1}{i} \frac{\partial}{\partial \eta} \log f(z, \zeta) .
\end{aligned}
$$

Therefore 


$$
\begin{aligned}
& \frac{P_{1}+P_{0}}{2}=-\frac{\partial}{\partial \zeta} \log f(z, \zeta) \\
& \frac{P_{1}-P_{0}}{2}=-\frac{\partial}{\partial \bar{\zeta}} \log f(z, \zeta) .
\end{aligned}
$$

If we now take the derivatives with respect to $z$ :

$$
\begin{aligned}
\frac{\partial}{\partial z}\left(\frac{P_{1}+P_{0}}{2}\right) d z & =-\frac{\partial^{2}}{\partial z \partial \zeta} \log f(z, \zeta) d z=\frac{\partial^{2} m(z, \zeta)}{\partial z \partial \zeta} d z \\
& =\frac{\partial^{2} N}{\partial z \partial \zeta} d z=-\pi L_{0}(z, \zeta) d z \\
\frac{\partial}{\partial z}\left(\frac{P_{1}-P_{0}}{2}\right) d z & =-\frac{\partial^{2}}{\partial z \partial \bar{\zeta}} \log f(z, \zeta) d z=\frac{\partial^{2} m(z, \zeta)}{\partial z \partial \bar{\zeta}} d z \\
& =\frac{\partial^{2} N}{\partial z \partial \bar{\zeta}} d z=\pi K_{0}(z, \bar{\zeta}) d z .
\end{aligned}
$$

But

$$
\begin{aligned}
& \frac{\partial^{2} N}{\partial z \partial \zeta}=\frac{\partial^{2} g(z, \zeta)}{\partial z \partial \zeta}+\frac{1}{4} \sum_{i=1}^{k-1} \sum_{j=1}^{k-1} \Pi_{i j} w_{i}^{\prime}(z) w_{j}^{\prime}(\zeta) \\
& \frac{\partial^{2} N}{\partial z \partial \bar{\zeta}}=-\frac{\partial^{2} g(z, \zeta)}{\partial z \partial \bar{\zeta}}-\frac{1}{4} \sum_{i=1}^{k-1} \sum_{j=1}^{k-1} \Pi_{i j} w_{i}^{\prime}(z) \overline{w_{j}^{\prime}(\zeta)}
\end{aligned}
$$

and

$$
\begin{aligned}
& K(z, \bar{\zeta}) d z=-\frac{1}{\pi} \frac{\partial^{2} g}{\partial z \partial \bar{\zeta}} d z \\
& L(z, \zeta) d z=-\frac{1}{\pi} \frac{\partial^{2} g}{\partial z \partial \zeta} d z
\end{aligned}
$$

Consequently:

$$
\begin{aligned}
& \frac{\partial}{\partial z}\left(\frac{P_{1}+P_{0}}{2}\right) d z=-\pi L(z, \zeta) d z+\frac{1}{4} \sum_{i=1}^{k-1} \sum_{j=1}^{k-1} \Pi_{i j} w_{i}^{\prime}(z) w_{j}^{\prime}(\zeta) d z \\
& \frac{\partial}{\partial z}\left(\frac{P_{1}-P_{0}}{2}\right) d z=-\pi K(z, \bar{\zeta}) d z+\frac{1}{4} \sum_{i=1}^{k-1} \sum_{j=1}^{k-1} \Pi_{i j} w_{i}^{\prime}(z) \overline{w_{j}^{\prime}(\zeta)} d z
\end{aligned}
$$

2G. We now shall study in a more detailed way the orthogonal complement of $\Gamma_{a e}$. We recall that

$$
\Gamma_{a}=\Gamma_{a s e}+\Gamma_{a m} .
$$

In our planar case, $\Gamma_{a s e}=\Gamma_{a e}$, and $\Gamma_{a}$ has then the decomposition

$$
\Gamma_{a}=\Gamma_{a e}+\Gamma_{a m} \text {. }
$$

2H. The following lemma is useful for the study of reproducing 
kernels.

LEMma. If $\Gamma_{a}=\Gamma_{1}+\Gamma_{2}+\cdots+\Gamma_{n}$, if there exists a reproducing kernel $k d z$ for $\Gamma_{a}$, and if $k d z$ has the decomposition

$$
k d z=\sum_{i=1}^{n} k_{i} d z \quad \text { where } \quad k_{i} d z \in \Gamma_{i}
$$

then $k_{i} d z$ is the reproducing kernel for $\Gamma_{i}$.

Proof. Let $a_{i} d z \in \Gamma_{i}$. By the reproducing property of $k d z$ on $\Gamma_{a}$, $\left(a_{i} d z, k d z\right)=a_{i}(\zeta)$. But

$$
\begin{aligned}
\left(a_{i} d z, k d z\right) & =\left(a_{i} d z, \sum_{j=1}^{n} k_{j} d z\right)=\sum_{j=1}^{n}\left(a_{i} d z, k_{j} d z\right) \\
& =\left(a_{i} d z, k_{i} d z\right)
\end{aligned}
$$

because

$$
\left(a_{i} d z, k_{j} d z\right)=0 \text { for } i \neq j
$$

This proves

$$
\left(a_{i} d z, k_{i} d z\right)=a_{i}(\zeta)
$$

2I. We shall prove a somewhat related proposition for the orthogonal kernel.

Lemma. Let $\Gamma_{a}=\Gamma_{1}+\Gamma_{2}+\cdots+\Gamma_{n}$.

Let $s d z$ be a singular differential which is in $\Gamma_{k}, 1 \leqq k \leqq n$ except for some analytic singularity $\theta$.

Let us suppose there exists an orthogonal kernel $h d z$ for $\Gamma_{a}$ corresponding to the singularity $\theta$.

If $h d z-s d z=\sum_{i=1}^{n} \mathfrak{h}_{i} d z$.

Then $s d z+\mathfrak{h}_{k} d z$ is the orthogonal kernel for $\Gamma_{k}$.

Proof. Let $a_{k} d z \in \Gamma_{k}$. hdz is orthogonal to $\Gamma_{a}$ hence:

$$
\left(a_{k} d z, h d z\right)=0=\left(a_{k} d z, s d z+\sum_{i=1}^{n} \mathfrak{h}_{i} d z\right)=\left(a_{k} d z, s d z+\mathfrak{h}_{k} d z\right) .
$$

But $s d z+\mathfrak{h}_{k} d z \in \Gamma_{k}$ except for the singularity, therefore

$$
s d z+\mathfrak{h}_{k} d z
$$

is the orthogonal kernel for $\Gamma_{k}$ corresponding to the singularity $\theta$.

Conversely, let us suppose we know the orthogonal kernel $s d z+$ $\mathfrak{h}_{k} d z$ for $\Gamma_{k}$, we can extend it to an orthogonal kernel for $\Gamma_{a}$. Let 
consequently $\Gamma_{a}=\Gamma_{k}+\Gamma_{l}$, and let us suppose there exists a finite basis for $\Gamma_{l}$. By the process of orthonormalization one can get an orthonormal basis

$$
e_{1} d z, e_{2} d z, \cdots, e_{m} d z \text { for } \Gamma_{l} \text {. }
$$

Any element of $\Gamma_{a}$ has the following expression:

$$
a d z=a_{k} d z+\sum_{i=1}^{m} \mu_{i} e_{i} d z
$$

Let us suppose that the corresponding orthogonal kernel for $\Gamma_{a}$ is

$$
h d z=s d z+\mathfrak{h}_{k} d z+\sum_{i=1}^{m} \nu_{i} e_{i} d z
$$

Then

$$
\begin{aligned}
(a d z, h d z)=0= & \left(a_{k} d z+\sum_{i=1}^{m} \mu_{i} e_{i} d z, s d z+\mathfrak{h}_{k} d z+\sum_{i=1}^{m} \nu_{i} e_{i} d z\right) \\
= & \left(a_{k} d z, s d z+\mathfrak{h}_{k} d z\right)+\left(a_{k} d z, \sum_{i=1}^{m} \nu_{i} e_{i} d z\right) \\
& +\left(\sum_{i=1}^{m} \mu_{i} e_{i} d z, s d z+\mathfrak{h}_{k} d z\right)+\left(\sum_{i=1}^{m} \mu_{i} e_{i} d z, \sum_{i=1}^{m} \nu_{i} e_{i} d z\right) \\
= & \sum_{i=1}^{m} \mu_{i}\left[\left(e_{i} d z, s d z+\mathfrak{h}_{k} d z\right)+\left(e_{i} d z, \sum_{i=1}^{m} \nu_{i} e_{i} d z\right)\right] \\
= & \sum_{i=1}^{m} \mu_{i}\left[\left(e_{i} d z, s d z\right)+\nu_{i}\left(e_{i} d z, e_{i} d z\right)\right] .
\end{aligned}
$$

This has to be true for all $\mu_{i}$, hence:

$$
\nu_{i}=-\left(e_{i} d z, s d z\right) \text {. }
$$

2J. We now shall apply the two preceeding lemmas to the construction of the reproducing kernels for the orthogonal complement of $\Gamma_{a e}$ in $\Gamma_{a}$, namely $\Gamma_{a m}$, and of the orthogonal kernel for $\Gamma_{a}$ corresponding to the singularity $d z /(z-\zeta)^{2}$. The method has an obvious extension to the construction of $m$ kernels and of orthogonal kernels corresponding to $d z /(z-\zeta)^{m+2}, m>0$.

2K. First let us construct the reproducing kernel $\hat{k}_{0}(z, \zeta) d z$ for $\Gamma_{a m}$. The analytic measures for the $k-1$ boundary contours $\beta_{i}$ form a basis for $\Gamma_{a m}$. Let $w_{i}^{\prime}(z) d z$ be the corresponding differentials. We wish to construct a linear combination

$$
\sum^{k-1} a_{i}(\zeta) w_{i}^{\prime}(z) d z
$$

which possesses the reproducing property on the elements of the basis: 


$$
\left(w_{j}^{\prime}(z) d z, \sum_{i=1}^{k-1} a_{i}(\zeta) w_{i}^{\prime}(z) d z\right)=w_{j}^{\prime}(\zeta)(j=1 \cdots k)
$$

or

$$
\sum_{i=1}^{k-1} \overline{a_{i}(\zeta)}\left(w_{j}^{\prime} d z, w_{i}^{\prime} d z\right)=w_{j}^{\prime}(\zeta)
$$

We have to compute

$$
\begin{aligned}
\left(w_{j}^{\prime} d z, w_{i}^{\prime} d z\right) & =\left(d \omega_{j}+i d \omega_{j}^{*}, d \omega_{i}+i d \omega_{i}^{*}\right)=2\left(d \omega_{j}, d \omega_{i}\right) \\
& =2 \int_{\bar{W}} d \omega_{j} d \omega_{i}^{*}=2 \int_{\beta} \omega_{j} d \omega_{i}^{*} \\
& =-2 \int_{\beta} \omega_{j} \frac{\partial \omega_{i}}{\partial n} d s=-2 \int_{\beta_{j}} \frac{\partial \omega_{i}}{\partial n} d s=4 \pi P_{i j} .
\end{aligned}
$$

Therefore:

$$
4 \pi \sum_{i=1}^{k-1} \overline{a_{i}(\zeta)} P_{i j}=w_{j}^{\prime}(\zeta)
$$

and

$$
\overline{a_{i}(\zeta)}=\frac{1}{4 \pi} \sum_{j=1}^{k-1} \Pi_{i j} w_{j}^{\prime}(\zeta)
$$

which shows that the reproducing kernel $\hat{k}_{0}(z, \zeta) d z$ for $\Gamma_{a m}$ is:

$$
\hat{k}_{0}(z, \zeta) d z=\frac{1}{4 \pi} \sum_{i=1}^{k-1} \sum_{j=1}^{k-1} \Pi_{i j} \overline{w_{j}^{\prime}}(\zeta) w_{i}^{\prime}(z) d z .
$$

2L. To find now the orthogonal kernel $\hat{h}(z, \zeta) d z$ corresponding to the singularity $d z /(z-\zeta)^{2}$ we look for an expression

$$
\hat{h}(z, \zeta) d z=\frac{d z}{(z-\zeta)^{2}}+\sum_{i=1}^{k-1} b_{i}(\zeta) w_{i}^{\prime}(z) d z .
$$

By definition, for $w_{j}^{\prime}(z) d z \in \Gamma_{a m}$

$$
\left(w_{j}^{\prime}(z) d z, \frac{d z}{(z-\zeta)^{2}}+\sum_{i=1}^{k-1} b_{i}(\zeta) w_{i}^{\prime}(z) d z\right)=0
$$

or

$$
\sum_{i=1}^{k-1} b_{i}\left(w_{j}^{\prime}(z) d z, w_{i}^{\prime}(z) d z\right)=-\left(w_{j}^{\prime}(z) d z, \frac{d z}{(z-\zeta)^{2}}\right)
$$

Now

$$
\left(w_{j}^{\prime}(z) d z, \frac{d z}{(z-\zeta)^{2}}\right)=-\frac{1}{i} \int_{\bar{W}} \frac{w_{j}^{\prime}(z)}{(\bar{z}-\bar{\zeta})^{2}} d z \overline{d z}
$$


Let $\alpha$ be the boundary of a circle of radius $r$ around $z=\zeta$. Then

$$
\begin{aligned}
\left(w_{j}^{\prime} d z, \frac{d z}{(z-\zeta)^{2}}\right) & =\lim _{r \rightarrow 0} \frac{1}{i} \int_{\beta-\alpha} \frac{w_{j}^{\prime}(z) d z}{(\bar{z}-\bar{\zeta})} \\
& =\frac{1}{i} \int_{\beta} \frac{w_{j}^{\prime}(z) d z}{(\bar{z}-\bar{\zeta})}-\lim _{r \rightarrow 0} \int_{\alpha} \frac{w_{j}^{\prime}(z) d z}{(\bar{z}-\bar{\zeta})} .
\end{aligned}
$$

The last integral has limit zero, because on $\alpha, \bar{z}-\bar{\zeta}=r^{2} /(z-\zeta)$. On $\beta$ however, $w_{j}^{\prime}(z)$ is imaginary. It follows then that on $\beta$

$$
w_{\jmath}^{\prime}(z) d z=-\overline{w_{j}^{\prime}(z) d z} .
$$

Therefore

$$
\left(w_{j}^{\prime} d z, \frac{d z}{(z-\zeta)^{2}}\right)=-\frac{1}{i} \int_{\beta} \frac{\overline{w_{j}^{\prime}(z) d z}}{(\bar{z}-\bar{\zeta})}=-\overline{i \int_{\beta} \frac{w_{j}^{\prime}(z) d z}{(z-\zeta)}} .
$$

The integral can be transferred to $\alpha$ hence

$$
\left(w_{\jmath}^{\prime} d z, \frac{d z}{(z-\zeta)^{2}}\right)=-\overline{i \int_{\alpha} \frac{w_{j}^{\prime}(z) d z}{z-\zeta}}=2 \pi \overline{w_{j}^{\prime}(\zeta)} .
$$

We find then that:

$$
\sum_{i=1}^{k-1} b_{i} P_{i j} 4 \pi=-2 \pi \overline{w_{j}^{\prime}(\zeta)}
$$

or

$$
b_{i}=-\frac{1}{2} \sum_{j=1}^{k-1} \Pi_{i j} \overline{w_{i}^{\prime}(\zeta)} .
$$

The orthogonal kernel has then the following expression:

$$
\hat{h}_{0}(z, \zeta) d z=\frac{d z}{(z-\zeta)^{2}}-\frac{1}{2} \sum_{i=1}^{k-1} \sum_{j=1}^{k-1} \Pi_{i j} \overline{w_{i}^{\prime}(\zeta)} w_{i}^{\prime}(z) d z .
$$

The results obtained in this section may be extended to open Riemann surfaces, following the method which will be outlined in the next chapter.

\section{Chapter IV. RIEMANN SURFACES OF NON-ZERO GENUS}

1. Kernels For $\Gamma_{a e}$. We shall extend in this paragraph our results about reproducing and orthogonal kernels to non-planar open Riemann surfaces. We shall first construct the reproducing kernel for $\Gamma_{a e}$.

1A. We recall the orthogonal decomposition:

$$
\Gamma_{a}=\Gamma_{a e}+\Gamma_{a s} .
$$


On an arbitrary surface $W$, the principal functions $p_{0}$ and $p_{1}$ have vanishing flux. Therefore the differentials $\partial p_{0} / \partial z$ and $\partial p_{1} / \partial z$ are analytic semi-exact. It follows that $(\partial / \partial z)\left(\left(p_{1}-p_{0}\right) / 2\right) d z \in \Gamma_{a s e}$. Therefore $-(1 / \pi)(\partial / d z)\left(\left(p_{1}-p_{0}\right) / 2\right) d z$ has a unique decomposition:

$$
-\frac{1}{\pi} \frac{\partial}{\partial z} \frac{\left(p_{1}-p_{0}\right)}{2} d z=\omega_{e}+\omega_{s}, \quad \text { where } \omega_{e} \in \Gamma_{a e}, \omega_{s} \in \Gamma_{a s} .
$$

Let us first consider a compact bordered surface $\bar{W}$. The differential $\omega_{s}$ has the same periods as $-(1 / \pi)(\partial / \partial z)\left(\left(p_{1}-p_{0}\right) / 2\right) d z$. The analytic Schottky differential $\omega_{s}$ may be written as:

$$
\omega_{s}=\phi_{1}+i \phi_{2}
$$

where $\phi_{1}$ and $\phi_{2}$ are analytic Schottky differentials which are real on the boundary $\beta$ of $W$. We shall prove the following result:

THEOREM. Let $p_{1}$ and $p_{0}$ be the principal functions on $\bar{W}$ corresponding to the singularity $1 /(z-\zeta)$. Then

$$
\omega_{e}=-\frac{1}{\pi} \frac{\partial}{\partial z} \frac{\left(p_{1}-p_{0}\right)}{2} d z-\omega_{s}
$$

is the reproducing kernel for $\Gamma_{a e}(\bar{W})$.

Proof. Let $a^{\prime}(z) d z \in \Gamma_{a e}$. We compute the inner product $\left(a^{\prime}(z) d z, \omega_{e}\right)$ :

$$
\begin{aligned}
\left(a^{\prime}(z) d z, \omega_{e}\right) & =i \int_{\bar{W}} a^{\prime}(z) d z \overline{\omega_{e}} \\
& =i \int_{\beta} a(z)\left[-\frac{1}{\pi} \frac{\partial \overline{\left(p_{1}-p_{0}\right)}}{2 z} d z-\overline{\left(\phi_{1}+i \phi_{2}\right)}\right] \\
& =i \int_{\beta} a(z)\left[\frac{1}{\pi} \frac{\partial}{\partial z} \frac{\left(p_{1}+p_{0}\right)}{2} d z-\left(\phi_{1}-i \phi_{2}\right)\right] .
\end{aligned}
$$

The integral may be transferred to the boundary $\alpha$ of a circle of radius $r$ around $z=\zeta$. We get:

$$
\left(a^{\prime}(z) d z, \omega_{e}\right)=i \int_{\alpha} a\left[\frac{1}{\pi} \frac{\partial}{\partial z} \frac{\left(p_{1}+p_{0}\right)}{2} d z-\left(\phi_{1}-i \phi_{2}\right)\right] .
$$

But in a neighborhood of $z=\zeta$,

$$
\frac{\partial}{\partial z} \frac{\left(p_{1}+p_{0}\right)}{2} d z=\frac{-d z}{2(z-\zeta)^{2}}+b(z) d z
$$

where $b(z) d z$ is analytic. Therefore

$$
\left(a^{\prime}(z) d z, \omega_{e}\right)=-i \int_{\alpha} \frac{a(z) d z}{2 \pi(z-\zeta)^{2}}+i \int_{\alpha} a(z) \frac{b(z)}{2 \pi} d z-i \int_{\alpha} a(z)\left(\phi_{1}-i \phi_{2}\right) .
$$


When $r$ tends to zero the last two integrals tend to zero. The first has value $a^{\prime}(\zeta)$. Therefore

$$
\left(a^{\prime}(z) d z, \omega_{e}\right)=a^{\prime}(\zeta),
$$

which completes the proof of the theorem.

1B. We describe now a procedure to construct $\omega_{e}$. We suppose that $-(1 / \pi)(\partial / \partial z)\left(\left(p_{1}-p_{0}\right) / 2\right) d z$ is known; in particular its periods can be computed. The differential $\omega_{s}$ is an analytic Schottky differential on $\bar{W}$. It can be extended to the double $\hat{W}$ of $\bar{W}$. The double $\hat{W}$ is a closed surface, on which one can construct a unique analytic Schottky differential $\omega_{s}^{\prime}$ with the same periods as $-(1 / \pi)(\partial / \partial z)\left(\left(p_{1}-p_{0}\right) / 2\right) d z$. The expression $-(1 / \pi)(\partial / \partial z)\left(\left(p_{1}-p_{0}\right) / 2\right) d z-\omega_{s}^{\prime}$ has no period at all. It is analytic exact, and by the uniqueness of the decomposition $\omega_{s}^{\prime}=\omega_{s}$. Knowing $\omega_{s}$, we get $\omega_{e}=-(1 / \pi)\left(\left(p_{1}-p_{0}\right) / 2\right) d z-\omega_{s}$, the reproducing kernel for $\Gamma_{a e}(\bar{W})$.

1C. An analogous procedure can be applied to obtain orthogonal kernels with singularity $d z /(z-\zeta)^{m+2}, m \geqq 0$. We shall show, for instance, how to get the kernel corresponding to $d z /(z-\zeta)^{2}$.

Accordingly, let us suppose there exists a differential $s d z$ analytic exact except for the singularity $d z /(z-\zeta)^{2}$ on $\bar{W}$. We shall use the following decomposition for $-2(\partial / \partial z)\left(p_{1}+p_{0}\right) / 2 d z$ :

$$
\begin{array}{r}
-2 \frac{\partial}{\partial z} \frac{\left(p_{1}+p_{0}\right)}{2} d z-s d z=\omega_{1 e}+\omega_{1 s} \text { with } \omega_{1 e} \in \Gamma_{a e}(\bar{W}) \\
\omega_{1 s} \in \Gamma_{a s}(\bar{W}) .
\end{array}
$$

Let $a^{\prime} d z \in \Gamma_{a e}(\bar{W}), \omega_{1 s}=\psi_{1}+i \psi_{2}$ where $\psi_{1}$ and $\psi_{2}$ are analytic Schottky differentials on $\bar{W}$, which are real on $\beta$. We show that $-2(\partial / \partial z)\left(\left(p_{1}+\right.\right.$ $\left.\left.p_{0}\right) / 2\right) d z-\omega_{1 s}$ is the orthogonal kernel for $\bar{W}$ corresponding to the singularity $d z /(z-\zeta)^{2}$. Thus:

$$
\begin{aligned}
& \left(a^{\prime} d z,-2 \frac{\partial}{\partial z} \frac{\left(p_{1}+p_{0}\right)}{2} d z-\omega_{1 s}\right) \\
& =\lim _{r \rightarrow 0} i \int_{\beta-\alpha} a\left[-2 \frac{\partial \frac{\left(p_{1}+p_{0}\right)}{\partial z} d z}{2}-\overline{\left(\psi_{1}+i \psi_{2}\right)}\right] \\
& =i \int_{\beta} a\left[2 \frac{\partial}{\partial z} \frac{\left(p_{1}-p_{0}\right)}{2} d z-\left(\psi_{1}-i \psi_{2}\right)\right] \\
& \quad-\lim _{r \rightarrow 0} \int_{\alpha}-a\left[\frac{\partial}{\partial z} \frac{\overline{\left(p_{1}+p_{0}\right)}}{2} d z+\overline{\left(\psi_{1}+i \psi_{2}\right)}\right] .
\end{aligned}
$$

Clearly, the last integral tends to zero when $r \rightarrow 0$. The first integral may be written as an inner product and we get: 


$$
\begin{aligned}
\left(a^{\prime} d z,-2\right. & \left.\frac{\partial}{\partial z} \frac{\left(p_{1}+p_{0}\right)}{2}-\omega_{1 s}\right) \\
& =\left(a^{\prime} d z, 2 \frac{\left.\bar{\partial} \frac{\left(p_{1}-p_{0}\right)}{\partial z} d z-\left(\psi_{1}-i \psi_{2}\right)\right)}{2}=0 .\right.
\end{aligned}
$$

To actually construct the kernel, it suffices to compute the periods of $-2(\partial / \partial z)\left(p_{1}+p_{0}\right) / 2$ and obtain, as in the case of the reproducing kernel, the analytic Schottky differential which exhibits the same periods on $\bar{W}$.

1D. We wish now to extend the preceding results to the case of open Riemann surfaces. Let $W$ be an arbitrary Riemann surface, $\left\{\Omega_{n}\right\}$ a canonical exhaustion of $W$. Each $\Omega_{n}$ is of finite genus and has a compact bordered closure.

Let $\Gamma_{a e}\left(\Omega_{n}\right)$ be the space of analytic differentials on $\Omega_{n}$; we call $k_{0 \Omega_{n}}(z, \zeta) d z$ the reproducing kernel for $\Gamma_{a e}\left(\Omega_{n}\right), h_{m \Omega_{n}}(z, \zeta) d z$ the orthogonal kernel for $\Gamma_{a e}\left(\Omega_{n}\right)$ corresponding to the singularity $d z /(z-\zeta)^{m+2}, m \geqq 0$. We prove the following proposition.

1E. THEOREM. If $W$ is not of class $\mathcal{O}_{A D}$, there exists a reproducing kernel $\check{k}_{0}(z, \zeta) d z$ for $\Gamma_{a e}(W)$.

Proof. Let $\Omega \subset \Omega^{\prime} \subset W$, when $\Omega$ and $\Omega^{\prime}$ are elements of $\left\{\Omega_{n}\right\}$. Let $\zeta \in \Omega$. We recall that a reproducing kernel is nonnegative at $z=\zeta$. Then:

$$
\left\|\check{k}_{0, \Omega^{\prime}} d z-\check{k}_{0, \Omega} d z\right\|_{\Omega}^{2}=\left\|\check{k}_{0 \Omega} d z\right\|_{\Omega}^{2}+\left\|\check{k}_{0, \Omega^{\prime}} d z\right\|_{\Omega}^{2}-2 \check{k}_{0, \Omega^{\prime}}(\zeta, \zeta)
$$

or

$$
\begin{aligned}
\left\|\check{k}_{0, \Omega^{\prime}} d z-\check{k}_{0 \Omega} d z\right\|_{\Omega}^{2} & \leqq\left\|\check{k}_{0 \Omega} d z\right\|_{\Omega}^{2}+\left\|\check{k}_{0, \Omega^{\prime}} d z\right\|_{\Omega^{\prime}}^{2}-2 \check{k}_{0 \Omega^{\prime}}(\zeta, \zeta) \\
& =\check{k}_{0, \Omega}(\zeta, \zeta)-\check{k}_{0, \Omega^{\prime}}(\zeta, \zeta) .
\end{aligned}
$$

Therefore the sequence $\left\{\check{k}_{0, \Omega_{n}}(\zeta, \zeta)\right\}$ is a nonincreasing sequence of nonnegative numbers. It has then a limit when $n \rightarrow \infty$. If $W \notin \mathcal{O}_{A D}$, this limit cannot be zero. In fact, if there exists on $W$ an analytic function $f(z)$ of bounded norm, then $f^{\prime}(z) d z \in \Gamma_{a e}(W)$ and, by Theorem $2 \mathrm{~A}$, Ch. II, if $f^{\prime}(\zeta) \neq 0$.

$$
\left\|\frac{k_{0 \Omega}(z, \zeta) d z}{\check{k}_{0 \Omega}(\zeta, \zeta)}\right\|_{\Omega}^{2} \leqq\left\|\frac{f^{\prime}(z) d z}{f^{\prime}(\zeta)}\right\|_{\Omega}^{2}
$$

or

$$
\frac{1}{k_{0 \Omega}(\zeta, \zeta)} \leqq\left\|\frac{f^{\prime}(z) d z}{f^{\prime}(\zeta)}\right\|_{\Omega}^{2}
$$

when $\Omega \rightarrow W$, the right hand side remains bounded. Hence the left 
hand side has to remain bounded too and

$$
\lim _{n \rightarrow \infty} \check{k}_{0 \Omega_{n}}(\zeta, \zeta) \neq 0 \text {. }
$$

But

$$
\left\|\check{k}_{0, \Omega^{\prime}} d z-\check{k}_{0, \Omega} d z\right\|_{\Omega^{\prime \prime}}^{2} \leqq\left\|\check{k}_{0, \Omega^{\prime}} d z-\check{k}_{0, \Omega} d z\right\|_{\Omega}^{2} \leqq \check{k}_{0 \Omega}(\zeta, \zeta)-\check{k}_{0, \Omega^{\prime}}(\zeta, \zeta) \text {. }
$$

where $\Omega^{\prime \prime}$ is fixed and $\Omega^{\prime \prime} \subset \Omega \subset \Omega^{\prime}$.

If $\Omega$ now tends to $W$, then

$$
\lim _{\Omega, \Omega^{\prime} \rightarrow W}\left\|\check{k}_{0 \Omega^{\prime}} d z-\check{k}_{0 \Omega} d z\right\|_{\Omega^{\prime \prime}}=0
$$

and therefore $\breve{k}_{0 \Omega} d z$ has a limit $\check{k}_{0} d z$ when $\Omega \rightarrow W$ and that limit is attained uniformly on every compact subset $E \subset W$.

Moreover, for each $\Omega \in\left\{\Omega_{n}\right\}$ the following decomposition holds:

$$
\check{k}_{0.2} d z=-\frac{\partial}{\pi \partial z}\left(\frac{p_{1}-p_{0}}{2}\right)_{\Omega} d z-\omega_{s \Omega} .
$$

The preceding equation shows that when $\Omega$ tends to $W, \omega_{s \Omega}$ has a limit such that

$$
\begin{aligned}
\lim _{\Omega \rightarrow W} \omega_{s \Omega} & =\lim _{\Omega \rightarrow W}\left(-\check{k}_{0 \Omega} d z\right)+\lim _{\Omega \rightarrow W}\left(-\frac{\partial}{\pi \partial z}\left(\frac{p_{1}-p_{0}}{2}\right)_{\Omega} d z\right) \\
& =-\check{k}_{0} d z-\frac{\partial}{\pi \partial z}\left(\frac{p_{1}-p_{0}}{2}\right)_{W} .
\end{aligned}
$$

It remains to show that $\check{k}_{0} d z$ has the reproducing property. For $a(z) d z \epsilon$ $\Gamma_{a e}(W)$ we form:

$$
\left(a(z) d z, \check{k}_{0} d z-\check{k}_{0 \Omega} d z\right)_{\Omega}=\left(a(z) d z, \check{k}_{0} d z\right)_{\Omega}-a(\zeta) .
$$

By Schwarz's inequality

$$
\left|\left(a(z) d z, \check{k}_{0} d z\right)_{\Omega}-a(\zeta)\right|^{2} \leqq\|a(z) d z \mid\|_{\Omega}^{2}\left\|\check{k}_{0} d z-\check{k}_{0 \Omega} d z\right\|_{\Omega}^{2} ;
$$

when $\Omega$ tends to $W$ :

$$
\lim _{\Omega \rightarrow W}\left|\left(a(z) d z, \check{k_{0}} d z\right)_{\Omega}-a(\zeta)\right|=0 .
$$

Therefore

$$
\left(a(z) d z, \check{k}_{0} d z\right)=a(\zeta)
$$

1F. An analogous proof can be used to show the existence of orthogonal kernels $\check{h}_{0 m} d z$ on $W$.

1G. We prove now that if $W \in \mathcal{O}_{A D}$, the reproducing kernel $\check{k}_{0 \Omega} d z$ 
vanishes as $\Omega \rightarrow W$. If not,

$$
\left\|\check{k}_{0} d z\right\|^{2}=\check{k}_{0}(\zeta, \zeta) \neq \infty
$$

and the preceding proof can be carried over. But then $\check{k_{0}} d z$ is the differential of an analytic function of finite Dirichlet norm, which contradicts the fact that $W \in \mathcal{O}_{A D}$.

\section{Kernels for $\Gamma_{a}$.}

2A. We shall use the following decomposition of $\Gamma_{a}$ :

$$
\Gamma_{a}=\Gamma_{a e}+\Gamma_{a s} .
$$

We have constructed a reproducing kernel for $\Gamma_{a e}$. To get the reproducing kernel for $\Gamma_{a}$, it remains to find the reproducing kernel for $\Gamma_{a s}$.

2B. Let $\Omega \in\left\{\Omega_{n}\right\}$. We consider the double $\hat{\Omega}$ of $\Omega$. On $\hat{\Omega}$ there exists a canonical homology basis $\left\{A_{i}, B_{i}\right\}$. One may define on $\hat{\Omega}$ analytic differentials $\alpha_{k}$ with period 1 on $A_{k}, 0$ on $A_{h}$ for $h \neq k$, and "symmetric" on $\hat{\Omega}$ in the sense that they are analytic Schottky differentials on $\Omega$.

These $\alpha_{k}=a_{k} d z$ form a basis for $\Gamma_{a s}(\Omega)$. They are linearly independent because their periods are linearly independent, and every $a(z) d z \in \Gamma_{a s}(\Omega)$ can be expressed as a linear combination of the $\alpha_{k}$ : if we subtract from $a(z) d z$ a linear combination of the $\alpha_{k}$ such that the difference has no $A_{h}$-periods, this difference can be extended by "symmetry" to $\hat{\Omega}$, and we would have on $\hat{\Omega}$ an analytic differential without $A_{h}$-period. Such a differential is necessarily zero.

2C. Let $N$ be the total number of $\alpha_{k}$. $N$ is equal to $g^{\prime}$, the genus of $\hat{\Omega}$. It depends on the genus $g$ and on the number of contours $c$ of $\Omega: \quad g^{\prime}=2 g+c-1$.

We form now a linear combination

$$
\sum_{i=1}^{N} b_{i} a_{i}(z) d z
$$

which has the reproducing property on $\Gamma_{a s}(\Omega)$. It suffices to show that the property is valid for the $\alpha_{k},(k=1 \cdots N)$. We want

$$
\left(a_{k}(z) d z, \sum_{i=1}^{N} b_{i}(\zeta) a_{i}(z) d z\right)_{\Omega}=a_{k}(\zeta)
$$

or

$$
\sum_{i=1}^{N} \bar{b}_{i}\left(a_{k} d z, a_{i} d z\right)_{\Omega}=a_{k}(\zeta)
$$


Because the $a_{i} d z$ are a basis, the matrix $\left(t_{k l}\right)$ such that

$$
\left(a_{k} d z, a_{l} d z\right)_{\Omega}=t_{k l}
$$

is non-singular.

We now may solve the system for the $b_{i}$

$$
\begin{gathered}
\sum_{i=1}^{N} \bar{b}_{i}(\zeta) t_{k i}=a_{k}(\zeta) \\
\overline{b_{\imath}(\zeta)}=\sum_{k=1}^{N} T_{i k} a_{k}(\zeta) \quad \text { where } \quad\left(T_{i k}\right)=\left(t_{k i}\right)^{-1}
\end{gathered}
$$

and we get the reproducing kernel:

$$
k_{0 . \Omega}^{\sharp}(z, \zeta) d z=\sum_{i=1}^{N} \sum_{k=1}^{N} \overline{T_{i k} a_{k}}(\zeta) a_{i}(z) d z .
$$

2D. By the method of the preceding section it is now easy to show that $k_{0}^{\sharp} d z=\lim _{\Omega \rightarrow W} k_{0 \Omega}^{\sharp}(z, \zeta) d z$ exists and possesses the reproducing property. We have constructed the kernel

$$
k_{0}(z, \zeta) d z=\hat{k}_{0}(z, \zeta) d z+k_{0}^{\sharp}(z, \zeta) d z
$$

which has the reproducing property on $\Gamma_{a}(W)$.

By an obvious modification of the preceding argument one may construct $m$ kernels $k_{m} d z$, and orthogonal kernels $h_{m} d z(m \geqq 0)$ for $\Gamma_{a}(W)$.

\section{BIBLIOGRAPHY}

1. L. Ahlfors, and L. Sario, Riemann surfaces, Princeton University Press, 1960.

2. S. Bergman, The kernel function and conformal mapping, Mathematical Surveys Number V, American Mathematical Society (1950).

3. L. Sario, A linear operator method on arbitrary Riemann surfaces, Trans. Amer. Math. Soc., 72 (1952), 281-295.

4. - Functionals on Riemann surfaces, Lectures on functions of a complex variable, the University of Michigan Press (1955).

5. M. Schiffer, Some recent developments in the theory of conformal mapping, Appendix to R. Courant, Dirichlet's principle, conformal mapping, and minimal surfaces, Interscience Publishers (1950), 249-265.

University of CAlifornia, Los ANGEles 



\title{
PACIFIC JOURNAL OF MATHEMATICS
}

\author{
EDITORS
}

Ralph S. Phillips

Stanford University

Stanford, California

M. G. Arsove

University of Washington

Seattle 5, Washington
A. L. Whiteman

University of Southern California Los Angeles 7, California

Lowell J. Paige

University of California

Los Angeles 24, California

\section{ASSOCIATE EDITORS}
E. F. BECKENBACH
D. DERRY
M. OHTSUKA
H. L. ROYDEN
E. SPANIER
E. G. STRAUS
T. M. CHERRY
F. WOLF

\section{SUPPORTING INSTITUTIONS}

\author{
UNIVERSITY OF BRITISH COLUMBIA \\ CALIFORNIA INSTITUTE OF TECHNOLOGY \\ UNIVERSITY OF CALIFORNIA \\ MONTANA STATE UNIVERSITY \\ UNIVERSITY OF NEVADA \\ NEW MEXICO STATE UNIVERSITY \\ OREGON STATE UNIVERSITY \\ UNIVERSITY OF OREGON \\ OSAKA UNIVERSITY \\ UNIVERSITY OF SOUTHERN CALIFORNIA
}

\author{
STANFORD UNIVERSITY \\ UNIVERSITY OF TOKYO \\ UNIVERSITY OF UTAH \\ WASHINGTON STATE UNIVERSITY \\ UNIVERSITY OF WASHINGTON \\ $*$
AMERICAN MATHEMATICAL SOCIETY \\ CALIFORNIA RESEARCH CORPORATION \\ SPACE TECHNOLOGY LABORATORIES \\ NAVAL ORDNANCE TEST STATION
}

Mathematical papers intended for publication in the Pacific Journal of Mathematics should be typewritten (double spaced), and the author should keep a complete copy. Manuscripts may be sent to any one of the four editors. All other communications to the editors should be addressed to the managing editor, L. J. Paige at the University of California, Los Angeles 24, California.

50 reprints per author of each article are furnished free of charge; additional copies may be obtained at cost in multiples of 50 .

The Pacific Journal of Mathematics is published quarterly, in March, June, September, and December. Effective with Volume 13 the price per volume (4 numbers) is $\$ 18.00$; single issues, $\$ 5.00$. Special price for current issues to individual faculty members of supporting institutions and to individual members of the American Mathematical Society: $\$ 8.00$ per volume; single issues $\$ 2.50$. Back numbers are available.

Subscriptions, orders for back numbers, and changes of address should be sent to Pacific Journal of Mathematics, 103 Highland Boulevard, Berkeley 8, California.

Printed at Kokusai Bunken Insatsusha (International Academic Printing Co., Ltd.), No. 6, 2-chome, Fujimi-cho, Chiyoda-ku, Tokyo, Japan.

PUBLISHED BY PACIFIC JOURNAL OF MATHEMATICS, A NON-PROFIT CORPORATION

The Supporting Institutions listed above contribute to the cost of publication of this Journal, but they are not owners or publishers and have no responsibility for its content or policies. 


\section{Pacific Journal of Mathematics}

\section{Vol. 12, No. $2 \quad$ February, 1962}

William George Bade and Robert S. Freeman, Closed extensions of the Laplace operator determined by a general class of boundary conditions . . . . . . . 395

William Browder and Edwin Spanier, H-spaces and duality ............. 411

Stewart S. Cairns, On permutations induced by linear value functions . . . . . . . 415

Frank Sydney Cater, On Hilbert space operators and operator roots of

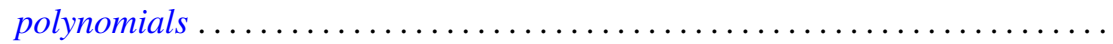

Stephen Urban Chase, Torsion-free modules over $K[x, y] \ldots \ldots \ldots \ldots \ldots \ldots \ldots 437$

Heron S. Collins, Remarks on affine semigroups . . . . . . . . . . . . . . . . 449

Peter Crawley, Direct decompositions with finite dimensional factors . . . . . . . 457

Richard Brian Darst, A continuity property for vector valued measurable

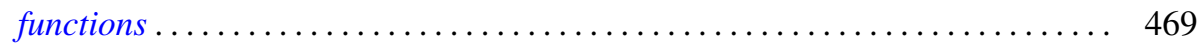

R. P. Dilworth, Abstract commutative ideal theory ................. 481

P. H. Doyle, III and John Gilbert Hocking, Continuously invertible spaces . . . . . . 499

Shaul Foguel, Markov processes with stationary measure . . . . . . . . . . . 505

Andrew Mattei Gleason, The abstract theorem of Cauchy-Weil ............ 511

Allan Brasted Gray, Jr., Normal subgroups of monomial groups . . . . . . . . . . 527

Melvin Henriksen and John Rolfe Isbell, Lattice-ordered rings and function

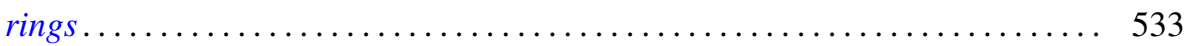

Amnon Jakimovski, Tauberian constants for the $[J, f(x)]$ transformations . ..... 567

Hubert Collings Kennedy, Group membership in semigroups . . . . . . . . . . . 577

Eleanor Killam, The spectrum and the radical in locally $m$-convex algebras ..... 581

Arthur H. Kruse, Completion of mathematical systems . . . . . . . . . . . . . 589

Magnus Lindberg, On two Tauberian remainder theorems ................ 607

Lionello A. Lombardi, A general solution of Tonelli's problem of the calculus of

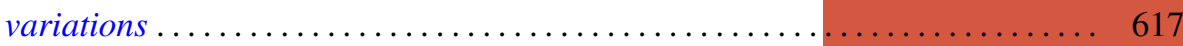

Marvin David Marcus and Morris Newman, The sum of the elements of the powers

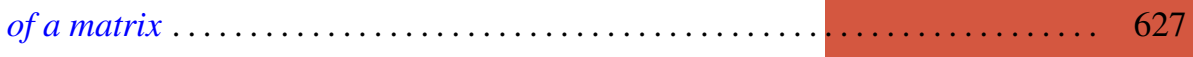

Michael Bahir Maschler, Derivatives of the harmonic measures in

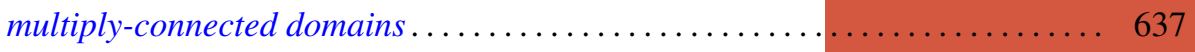

Deane Montgomery and Hans Samelson, On the action of $\mathrm{SO}(3)$ on $S^{n} \ldots \ldots \ldots 649$

J. Barros-Neto, Analytic composition kernels on Lie groups . . . . . . . . . . . . 661

Mario Petrich, Semicharacters of the Cartesian product of two semigroups ...... 679

John Sydney Pym, Idempotent measures on semigroups . . . . . . . . . . . . 685

K. Rogers and Ernst Gabor Straus, A special class of matrices . . . . . . . . . . . . 699

U. Shukla, On the projective cover of a module and related results . . . . . . . . . 709

Don Harrell Tucker, An existence theorem for a Goursat problem . . . . . . . . . . . 719

George Gustave Weill, Reproducing kernels and orthogonal kernels for analytic

differentials on Riemann surfaces ......................... 729

George Gustave Weill, Capacity differentials on open Riemann surfaces ........ 769

G. K. White, Iterations of generalized Euler functions . . . . . . . . . . . . . 777

Adil Mohamed Yaqub, On certain finite rings and ring-logics . . . . . . . . . 785 